\title{
Algebraic aspects of increasing subsequences
}

\author{
Jinho Baik* \\ Courant Institute of Mathematical Sciences, New York \\ baik@cims.nyu.edu \\ Eric M. Rains \\ AT\&T Research, New Jersey \\ rains@research.att.com
}

September 21, 2000

\begin{abstract}
We present a number of results relating partial Cauchy-Littlewood sums, integrals over the compact classical groups, and increasing subsequences of permutations. These include: integral formulae for the distribution of the longest increasing subsequence of a random involution with constrained number of fixed points; new formulae for partial Cauchy-Littlewood sums, as well as new proofs of old formulae; relations of these expressions to orthogonal polynomials on the unit circle; and explicit bases for invariant spaces of the classical groups, together with appropriate generalizations of the straightening algorithm.
\end{abstract}

\section{Introduction}

Consider the following two identities:

$$
\begin{aligned}
\sum_{\lambda} s_{\lambda}\left(x_{1}, x_{2}, \ldots\right) s_{\lambda}\left(y_{1}, y_{2}, \ldots\right) & =\prod_{i, j}\left(1-x_{i} y_{j}\right)^{-1} \\
\lim _{l \rightarrow \infty} \mathrm{E}_{U \in U(l)} \prod_{i} \operatorname{det}\left(1-x_{i} U\right)^{-1} \prod_{i} \operatorname{det}\left(1-y_{i} U^{-1}\right)^{-1} & =\prod_{i, j}\left(1-x_{i} y_{j}\right)^{-1} .
\end{aligned}
$$

The first of these is the well-known identity of Cauchy ([29]). The second is a formal analogue of the Szegö limit theorem, equivalent to a theorem of 10 . Since the right-hand sides are the same, we also have a third identity:

$$
\sum_{\lambda} s_{\lambda}\left(x_{1}, x_{2}, \ldots\right) s_{\lambda}\left(y_{1}, y_{2}, \ldots\right)=\lim _{l \rightarrow \infty} \mathrm{E}_{U \in U(l)} \prod_{i} \operatorname{det}\left(1-x_{i} U\right)^{-1} \prod_{i} \operatorname{det}\left(1-y_{i} U^{-1}\right)^{-1} .
$$

Our object of study in the present work is generalizations of these three identities.

*Address after Sep. 1, 1999 : Princeton University and Institite for Advanced Study, New Jersey 
Our generalizations take two forms. One is to remove the limit $l \rightarrow \infty$. As we shall see (section 5), in order to preserve (0.3), we must then restrict the sum over partitions to involve only partitions with at most $l$ parts. It is here that increasing subsequences appear: in order to rescue equations (0.1) and (0.2), we must replace the common right-hand side with a generating function counting objects (reducing to permutations in an appropriate limit) without long increasing subsequences. In the case of (0.1), the connection is via the Robinson-Schensted-Knuth correspondence, with its well-known connections to increasing subsequences. It turns out that there is also a direct connection for (0.2), in terms of the invariant theory of the unitary group. In particular, this gives a direct (and essentially elementary) proof of the known connection between unitary group integrals and increasing subsequences ([33]), as well as of the new connections given here.

The other way in which we generalize these identities is to replace the unitary group $U(l)$ by one of four other groups, including the orthogonal and symplectic groups. In terms of permutations, this corresponds to considering involutions (in two ways), signed permutations, and signed involutions, in addition to the original case of permutations; each of these conditions can be described as a particular type of symmetry condition. In each case, we obtain analogues of the finite $l$ versions of equations (0.1), (0.2), and (0.3), together with increasing subsequence interpretations.

\section{Guide to main results}

One of the classical models used to analyze increasing subsequences is the Poisson model: one generates a random subset of the unit square via a Poisson process, then associates a permutation to this subset in a canonical way (the order of the $y$ coordinates relative to the $x$ coordinates). The five symmetry types correspond to the five subgroups of $\mathbb{Z}_{2} \times \mathbb{Z}_{2}$ (acting on the unit square via diagonal reflections); one insists that the random subset be preserved by the appropriate group. It turns out that each symmetry type is naturally associated with a certain compact Lie group, determined as a fixed subgroup via an appropriate action of $\mathbb{Z}_{2} \times \mathbb{Z}_{2}$ on the unitary group. Our first main result (Theorem 1.2) then states that the (exact) distribution of the (length of the) longest increasing subsequence of a random permutation of a given symmetry type is given by the moments of the trace of a random element of the corresponding Lie group.

By the Schensted correspondence, each of the five cases of Theorem 1.2 can be viewed as expressing an integral over one of the five groups as an appropriate sum over partitions. Each such identity specializes an appropriate Schur function identity (Theorem 5.2 and Corollary 5.3). For the three symmetry types with diagonal symmetry, this can be further generalized (essentially allowing points on the diagonal of symmetry); thus, for instance, if $f(\lambda)$ is the number of odd parts and $\ell(\lambda)$ is the number of parts of $\lambda$, then (Theorem 5.6)

$$
\sum_{\ell(\lambda) \leq l} \alpha^{f(\lambda)} s_{\lambda}(x)=E_{U \in O(l)} \operatorname{det}(1+\alpha U) \prod_{j} \operatorname{det}\left(1-x_{j} U\right)^{-1} .
$$

For a quite general class of specializations (corresponding to "super-Schur" or "hook Schur" functions), these Schur function sums have combinatorial interpretations in terms of increasing subsequences of multisets.

That is, for each symmetry type and each specialization (subject to convergence conditions), we construct a random multiset and a notion of increasing subsequence such that (Theorem 7.1) the normalized Schur function sum gives the distribution of the longest increasing subsequence. These random multiset models generalize Johansson's 2D random growth model [24]. 
Putting these together, we obtain a connection between integrals and increasing subsequences of multisets. In each case, the identity states that the dimension of a certain space of invariants is given by counting a certain collection of multisets without long increasing subsequences. For the three classical groups, we give direct proofs of this fact by producing an explicit basis of the invariants indexed by the appropriate multisets. Thus, for instance (Theorem 8.2), the centralizer algebra of the $n$th tensor power representation of $U(l)$ has basis given by permutations of length $n$ with no increasing subsequence of length greater than $l$. More generally (Theorem 8.4), the space of simultaneous multilinear invariants of a collection of symmetric and antisymmetric, covariant and contravariant tensors is explicitly indexed by multisets without long increasing subsequences (generalizing the classical straightening algorithm). Corresponding results hold for the orthogonal (Theorems 8.5 and 8.6) and symplectic (Theorems 8.7 and 8.8) groups.

The remaining collection of results is of lesser interest combinatorially, but is crucial to our asymptotic analysis in [2]. The key step in the analysis is to express the integrals of interest in terms of orthogonal polynomials on the unit circle. This is done for the classical groups in Theorem 2.3 (the remaining two groups reduce to the unitary group); for the unitary group the connection is immediate, while for the orthogonal group one must pass through orthogonal polynomials on $[-1,1]$. We also give a number of results indicating how certain modifications to the integrand affect the integral. As a consequence, we find (Corollary 4.3) that for each of the five natural Poisson models, the longest increasing subsequence distribution can be expressed in terms of the same family of orthogonal polynomials. In the sequel to this paper [2], we determine the asymptotics of such polynomials via the Riemann-Hilbert method, and thus obtain the limiting longest increasing subsequence distribution for each of the five Poisson models as well as for the de-Poissonized versions (random symmetric permutations). The results are expressed in terms of the solution to the Painlevé II equation, thus connecting to random matrix theory. Further related asymptotic work can be found in [3], [1], [32].

\section{Outline}

Section 1 introduces the five symmetry types and their associated groups. In particular, we express the (exact) distribution of the longest increasing subsequence of a random permutation of a given symmetry type as an integral over the corresponding group. Section 2 expresses these integrals as determinants of Bessel functions related to orthogonal polynomials; this relation is given in section 3 .

Section 1 describes the extension of the integrals to include the cases when fixed points are allowed. In order to prove the formulae of section 1 , section 5 uses representation-theoretic arguments to deduce integral representations of partial Cauchy-Littlewood sums, at which point the theory of symmetric functions can be applied. Section 6 briefly discusses alternate proofs of those formulae based on intermediate pfaffian forms.

Section 7 uses a generalized Robinson-Schensted-Knuth correspondence from [0] to relate the partial CauchyLittlewood sums to increasing subsequences of certain distributions of random multisets.

Finally, section 8 shows that there is an intimate connection between increasing subsequences and invariants of the classical groups. Indeed, all of the integrals for which we give increasing subsequence interpretations also can be interpreted as dimensions of certain spaces of invariants. We give direct, elementary, proofs of

these identities, by constructing bases of invariants explicitly indexed by multisets with restricted increasing subsequenc length. In the process, we obtain a generalization of the straightening algorithm of invariant theory, as well as analogues for the orthogonal and symplectic groups. We also discuss extensions to quantum groups 
and supergroups.

Notation We refer the reader to [29] for notation and introduction to symmetric functions. In other notation, if $G$ is a compact group, we use

$$
\mathrm{E}_{U \in G} f(U)
$$

to denote the integral of $f(U)$ with respect to the (normalized) Haar measure on $G$. In other words, this is the expected value of $f$ evaluated at a uniform random element of $G$. When $G$ is the orthogonal group, we will occasionally need to consider the two components of $G$. Thus we will write

$$
\mathrm{E}_{U \in O^{ \pm}(l)} f(U)
$$

to denote the integral of $f$ over the coset of $O(l)$ of determinant \pm 1 . In particular, we have:

$$
\mathrm{E}_{U \in O^{ \pm}(l)} f(U)=\frac{1}{2} \mathrm{E}_{U \in O(l)}(1 \pm \operatorname{det}(U)) f(U) .
$$

Acknowledgments. We would like to acknowledge the following people for helpful discussions: Kurt Johansson for telling us about the processes generalized in section 7, Richard Stanley for telling us about the references for that section, Peter Shor for spotting flaws in earlier versions of the algorithms of section 8, and Christian Krattenthaler for helpful comments on section 5. We would also like to thank Jeff Lagarias, Andrew Odlyzko, and Neil Sloane for helpful comments and enthusiasm. The work of the first author was supported in part by a Sloan Doctoral Foundation Fellowship.

\section{Symmetrized increasing subsequence problems}

One of the standard models used in analyzing the usual increasing subsequence problem is defined as follows. We say that a collection of $k$ points in the unit square is "increasing" if for any two points $\left(x_{1}, y_{1}\right)$ and $\left(x_{2}, y_{2}\right)$, either $x_{1}<x_{2}$ and $y_{1}<y_{2}$ or $x_{1}>x_{2}$ and $y_{1}>y_{2}$. One can ask, then, for the distribution of the size of the largest increasing subset of $n$ points chosen uniformly and independently from the unit square. It is not too difficult to see that this is the same as the distribution of the longest increasing subsequence of a random permutation; indeed, we can associate a (uniformly distributed) permutation to a given collection of points by using the relative order of the $y$ coordinates after sorting along the $x$ coordinates. Also of interest is the Poissonized analogue, in which new points are occasionally added in such a way that the number of points at time $\lambda$ is Poisson with parameter $\lambda$.

One way to generalize this model is to impose a symmetry condition on the set of points. The square has 8 symmetries; if we insist that the symmetry preserve increasing collections, we obtain a group $H$ of 4 elements, generated by the reflections through the main diagonals. Thus there are 5 possible symmetry conditions we can impose (including the trivial condition), which we will denote by the symbols $\square, \nabla, \nabla$, $\nabla$, and $\nabla$, with associated groups $H_{\square}, H_{\square}, H_{\square}, H_{\square}$, and $H_{\square}$. (The symbol indicates the point/line(s) of reflection) We will also use the symbol $\circledast$ to denote an arbitrary choice of the five possibilities. 
Definition 1. We define

$$
p_{n l}^{\circledast}
$$

to be the probability that, if $n$ points are chosen uniformly at random in the unit square, then the set $\Sigma$ consisting of the images of those points under $H_{\circledast}$ contains no increasing subset with more than $l$ points. We also define a function

$$
Q_{l}^{\circledast}(\lambda)=e^{-\lambda} \sum_{0 \leq n} \frac{\lambda^{n} p_{n l}^{\circledast}}{n !} .
$$

The function $Q_{l}^{\circledast}(\lambda)$ corresponds to the natural Poisson model; $Q_{l}^{\circledast}(\lambda)$ is the probability that the largest increasing subset at time $\lambda$ has size at most $l$, and $1-Q_{l}^{\circledast}(\lambda)$ is the distribution function of the time at which an increasing subset of size $l+1$ first appears. In the sequel, however, it will turn out to be convenient to use a different time scale; we thus define

$$
P_{l}^{\circledast}(t)=Q_{l}^{\circledast}\left(a_{\circledast} t^{2} / 2\right),
$$

where $a_{\square}=a_{\square}=1, a_{\square}=a_{\square}=2$, and $a_{\square}=4$. (Here $a_{\circledast}$ is the number of Young tableaux associated to an element of $S_{n}^{\circledast}$ (q.v.))

If we map the set of points to a permutation, we obtain a permutation uniformly distributed from an appropriate ensemble. To be precise, define the involution $\iota \in S_{n}$ by $x \mapsto n+1-x$. Then define an ensemble $S_{n}^{\circledast}$ for each symmetry type as follows:

$$
\begin{aligned}
& S_{n}^{\square}=S_{n} \\
& S_{n}^{\square}=\left\{\pi \in S_{2 n}: \pi=\pi^{-1}, \pi(x) \neq x\right\} \\
& S_{n}^{\square}=\left\{\pi \in S_{2 n}: \pi=\iota \pi^{-1} \iota, \pi(x) \neq \iota(x)\right\} \\
& S_{n}^{\square}=\left\{\pi \in S_{2 n}: \pi=\iota \pi \iota\right\} \\
& S_{n}^{\square}=\left\{\pi \in S_{4 n}: \pi=\pi^{-1}, \pi=\iota \pi^{-1} \iota, \pi(x) \neq x, \iota(x)\right\} .
\end{aligned}
$$

Lemma 1.1. If a set $\Sigma$ is chosen as above with symmetry $\circledast$, then with probability 1 , the associated permutation is well-defined and is uniformly distributed from $S_{n}^{\circledast}$.

This motivates the further definition

$$
f_{n l}^{\circledast}=\left|S_{n}^{\circledast}\right| p_{n l}^{\circledast} .
$$

That is, $f_{n l}^{\circledast}$ is the number of elements of $S_{n}^{\circledast}$ with no increasing subsequence of length greater than $l$.

It is straightforward to compute $\left|S_{n}^{\circledast}\right|$ for each case:

$$
\begin{gathered}
\left|S_{n}^{\square}\right|=n ! \\
\left|S_{n}^{\square}\right|=\left|S_{n}^{\triangleright}\right|=\frac{(2 n) !}{2^{n} n !}
\end{gathered}
$$




$$
\begin{aligned}
& \left|S_{n}^{\square}\right|=2^{n} n ! \\
& \left|S_{n}^{\bowtie}\right|=\frac{(2 n) !}{n !} .
\end{aligned}
$$

Note that

$$
P_{l}^{\circledast}(t)=e^{-a_{\circledast} t^{2} / 2} \sum_{0 \leq n} f_{n l}^{\circledast} \frac{t^{2 n}}{n ! n !}
$$

for $\square$ and $\square$, and

$$
P_{l}^{\circledast}(t)=e^{-a_{\circledast} t^{2} / 2} \sum_{0 \leq n} f_{n l}^{\circledast} \frac{t^{2 n}}{(2 n) !}
$$

for $\square, \nabla$ and $\nabla$.

A major reason for considering the above problems is the following:

Theorem 1.2. Fix an integer $l>0$. Map $H$ into $\operatorname{Aut}(U(2 l))$ by

$$
/ \mapsto\left(U \mapsto\left(U^{t}\right)^{-1}\right) \quad \text { and } \quad \backslash \mapsto\left(U \mapsto-J\left(U^{t}\right)^{-1} J\right)
$$

where $J=\left(\begin{array}{cc}0 & -I_{l} \\ I_{l} & 0\end{array}\right)$. Let $U^{\circledast}(2 l)$ be the subgroup of $U(2 l)$ fixed by the corresponding automorphisms. Then

$$
f_{n(2 l)}^{\circledast}=\mathrm{E}_{U \in U \circledast(2 l)}\left(|\operatorname{Tr}(U)|^{2 n}\right) .
$$

Before giving the proof, it will be helpful to list the groups $U^{\circledast}(2 l)$ :

$$
\begin{aligned}
& U^{\square}(2 l)=U(2 l) \\
& U^{\square}(2 l)=O(2 l) \\
& U^{\square}(2 l)=S p(2 l) \\
& U^{\square}(2 l) \cong U(l) \times U(l) \\
& U^{\bigotimes}(2 l)=O(2 l) \cap S p(2 l) \cong U(l) .
\end{aligned}
$$

The last instance is the image of the fundamental representation of $U(l)$ as a $2 l \times 2 l$ real matrix, and thus corresponds to the direct sum of the fundamental representation and its conjugate.

Proof. The cases $\square, \square$, and $\square$ are given in [33]; more precisely, $\square$ is given there as Theorem 1.1, while $\square$ and $\nabla$ are given in Theorem 3.4. (Note that if $\pi \in S_{n}^{\triangleright}$, then $\pi \iota$ is a fixed-point-free involution with decreasing subsequences corresponding to the increasing subsequence of $\pi$.) We also give new, elementary, proofs below (Theorems 8.2, 8.5, and 8.7).

It remains to consider $\square$ and $\nabla$. Via the Robinson-Schensted correspondence (see [26], section 5.1.4, for an excellent introduction), we can associate a pair $(P, Q)$ of Young tableaux of the same shape to $\pi \in S_{n}^{\square}$, satisfying the relations $P^{S}=P, Q^{S}=Q$, where $S$ is the duality operation ("evacuation") of Schützenberger (ibid.). But there is a bijective correspondence between self-dual tableaux and domino tableaux (see, e.g., 42]), and further 
from domino tableaux to pairs of ordinary tableaux with disjoint content, and with shape determined only by the shape of the domino tableau ([37]). Thus we have associated four Young tableaux $\left(P_{1}, P_{2}, Q_{1}, Q_{2}\right)$, where $P_{1}$ and $Q_{1}$ have the same shape, $P_{2}, Q_{2}$ have the same shape, $P_{1}$ and $P_{2}$ have disjoint content, and $Q_{1}$ and $Q_{2}$ have disjoint content. This corresponds to a choice of $0 \leq m \leq n$, independent choices of two subsets of size $m$ of $[1,2, \ldots n]$, and independent choices of $\pi_{1}$ and $\pi_{2}$ of length $m$ and $n-m$. Furthermore, the longest increasing subsequence of $\pi$ has length at most $2 l$ precisely when the longest increasing subsequences of $\pi_{1}$ and $\pi_{2}$ are of length at most $l$. Putting this together, we find

$$
f_{n(2 l)}^{\square}=\sum_{0 \leq m \leq n}\left(\begin{array}{c}
n \\
m
\end{array}\right)^{2} f_{m l}^{\square} f_{(n-m) l}^{\square} .
$$

On the other hand, the integral formula simplifies as follows:

$$
\begin{aligned}
& \mathrm{E}_{U \in U} \varpi(2 l) \\
&\left(|\operatorname{Tr}(U)|^{2 n}\right)=\mathrm{E}_{U_{1} \in U(l), U_{2} \in U(l)}\left(\left|\operatorname{Tr}\left(U_{1}\right)+\operatorname{Tr}\left(U_{2}\right)\right|^{2 n}\right) \\
&=\mathrm{E}_{U_{1} \in U(l), U_{2} \in U(l)}\left(\left|\left(\operatorname{Tr}\left(U_{1}\right)+\operatorname{Tr}\left(U_{2}\right)\right)^{n}\right|^{2}\right) \\
&=\mathrm{E}_{U_{1} \in U(l), U_{2} \in U(l)}\left(\left|\sum_{0 \leq m \leq n}\left(\begin{array}{c}
n \\
m
\end{array}\right) \operatorname{Tr}\left(U_{1}\right)^{m} \operatorname{Tr}\left(U_{2}\right)^{n-m}\right|^{2}\right) \\
&=\sum_{0 \leq m \leq n}\left(\begin{array}{c}
n \\
m
\end{array}\right)^{2} f_{m l}^{\square} f_{(n-m) l}^{\square} \\
&=f_{n(2 l)}^{\square} .
\end{aligned}
$$

Similarly, an element $\pi \in S_{n}^{\bigotimes}$ corresponds to a pair of Young tableaux of the same shape with disjoint content, and thus

$$
f_{n(2 l)}^{\bigotimes}=\left(\begin{array}{c}
2 n \\
n
\end{array}\right) f_{n l}^{\square} .
$$

On the other hand,

$$
\begin{aligned}
\mathrm{E}_{U \in U^{\bigotimes}(2 l)}\left(|\operatorname{Tr}(U)|^{2 n}\right) & =\mathrm{E}_{U \in U(l)}\left((\operatorname{Tr}(U)+\overline{\operatorname{Tr}(U)})^{2 n}\right) \\
& =\sum_{0 \leq m \leq 2 n}\left(\begin{array}{c}
2 n \\
m
\end{array}\right) \mathrm{E}_{U \in U(l)}\left(\operatorname{Tr}(U)^{m} \overline{\operatorname{Tr}(U)}^{2 n-m}\right) \\
& =\left(\begin{array}{c}
2 n \\
n
\end{array}\right) \mathrm{E}_{U \in U(l)}\left(|\operatorname{Tr}(U)|^{2 n}\right) .
\end{aligned}
$$

It ought to be possible to give a more uniform proof of this result; the results of Section 8 (q.v.) may be relevant to this goal.

There is an analogue of Theorem 1.2 in which $2 l$ is replaced by $2 l+1$ :

Theorem 1.3. For any $n, l \geq 0$,

$$
\begin{aligned}
& f_{n(2 l+1)}^{\square}=\mathrm{E}_{U \in U(2 l+1)}|\operatorname{Tr}(U)|^{2 n} \\
& f_{n(2 l+1)}^{\square}=\mathrm{E}_{U \in O(2 l+1)}|\operatorname{Tr}(U)|^{2 n} \\
& f_{n(2 l+1)}^{\square}=\mathrm{E}_{U \in U(l) \oplus U(l+1)}|\operatorname{Tr}(U)|^{2 n},
\end{aligned}
$$


while

$$
\begin{aligned}
& f_{n(2 l+1)}^{\square}=f_{n(2 l)}^{\square} \\
& f_{n(2 l+1)}^{\square}=f_{n(2 l)}^{\square} .
\end{aligned}
$$

Also, we have the following corollary for $\square$ and $\bowtie$ :

Corollary 1.4. For any $n, l \geq 0$,

$$
\begin{aligned}
P_{2 l}^{\square}(t) & =P_{l}^{\square}(t)^{2} \\
P_{2 l+1}^{\square}(t) & =P_{l}^{\square}(t) P_{l+1}^{\square}(t) \\
P_{2 l}^{\square}(t) & =P_{l}^{\square}(t) .
\end{aligned}
$$

And for $\square, \nabla$, and $\nabla$, we have

Corollary 1.5. For any $n, l \geq 0$,

$$
\begin{aligned}
& P_{l}^{\square}(t)=e^{-t^{2} / 2} \mathrm{E}_{U \in O(l)} \exp (t \operatorname{Tr}(U)) \\
& P_{2 l}^{\square}(t)=e^{-t^{2} / 2} \mathrm{E}_{U \in S p(2 l)} \exp (t \operatorname{Tr}(U)) \\
& P_{2 l}^{\bigotimes}(t)=e^{-t^{2}} \mathrm{E}_{U \in U^{\bigotimes}(2 l)} \exp (t \operatorname{Tr}(U)) .
\end{aligned}
$$

Proof. For $\square$, we have

$$
e^{t^{2} / 2} P_{l}^{\square}(t)=\sum_{0 \leq n} \frac{t^{2 n}}{(2 n) !} \mathrm{E}_{U \in O(l)}\left(\operatorname{Tr}(U)^{2 n}\right)
$$

But $\mathrm{E}_{U \in O(l)} \operatorname{Tr}(U)^{n}=0$ for $n$ odd, so this is

$$
\mathrm{E}_{U \in O(l)} \exp (t \operatorname{Tr}(U))
$$

as required. The calculations for $\nabla$ and $\otimes$ are analogous.

Remark. In particular, we see that the formula (1.42) which was derived in $\sqrt{30}$ as an expression for $\square$ is really most naturally interpreted in $\otimes$ terms.

As an aside, we observe that if we remove the condition that the symmetries under consideration preserve increasing sets, but insist that the corresponding sets should still give permutations, there is one further type of symmetry allowed, namely rotation by 90 degrees. In terms of permutations, this is the set

$$
S_{n}^{\circ}=\left\{\pi \in S_{4 n}: \pi^{2}=\iota\right\} \quad\left|S_{n}^{\circ}\right|=(2 n) ! / n !
$$

Such permutations correspond to pairs of tableaux $\left(P, Q^{t}\right)$ with $n$ elements such that $P$ and $Q$ have the same shape and disjoint content. It follows that the length $l$ of the longest increasing subsequence from this set has the same distribution as $\max \left(2 l^{+}(\pi), 2 l^{-}(\pi)-1\right)$, where $\pi$ is randomly chosen from $S_{n}, l^{+}(\pi)$ is the increasing subsequence length of $\pi$, and $l^{-}(\pi)$ is the decreasing subsequence length of $\pi$. In particular, the bound of Erdos 
and Szekeres implies that $f_{n l}^{\circ}=0$ for $n>l^{2}$, and thus no integral formula à la Theorem 1.2 can exist for this case. There is a determinant formula, however, which can be obtained from the following symmetric function identity:

$$
\sum_{\substack{\ell(\lambda) \leq l^{+} \\
\ell\left(\lambda^{\prime}\right) \leq l^{-}}} s_{\lambda}(x) s_{\lambda^{\prime}}(y)=\operatorname{det}\left(\begin{array}{c}
\left(h_{j-i}(x)\right)_{0 \leq i<l^{+}, 0 \leq j<l^{+}+l^{-}} \\
\left.\left((-1)^{l^{+}+i-j} h_{l^{+}+i-j}(y)\right)_{0 \leq i<l^{-}, 0 \leq j<l^{+}+l^{-}}\right)
\end{array}\right) .
$$

One can either derive this via the approach in [16], or simply use the Jacobi-Trudi identity together with matrix manipulation as in 13. This then gives the following formulae:

$$
\begin{gathered}
\sum_{0 \leq n} f_{n(2 l)}^{\circ} \frac{t^{2 n}}{(2 n) !}=\operatorname{det}\left(\begin{array}{c}
\left(\frac{t^{j-i}}{(j-i) !}\right)_{0 \leq i<l, 0 \leq j<2 l} \\
\left(\frac{(-t)^{l+i-j}}{(l+i-j) !}\right)_{0 \leq i<l, 0 \leq j<2 l}
\end{array}\right) \\
\sum_{0 \leq n} f_{n(2 l+1)}^{\circ} \frac{t^{2 n}}{(2 n) !}=\operatorname{det}\left(\begin{array}{c}
\left(\frac{t^{j-i}}{(j-1) !}\right)_{0 \leq i<l, 0 \leq j<2 l+1} \\
\left(\frac{(-t)^{l+i-j}}{(l+i-j) !}\right)_{0 \leq i<l+1,0 \leq j<2 l+1}
\end{array}\right) .
\end{gathered}
$$

It is not clear how to obtain asymptotic information from the formulae, however, so we will not discuss this case further. (The techniques of [6] may be applicable, however.)

\section{Determinantal formulae}

It turns out that each of the formulae of Corollary 1.5 can be expressed in terms of a Toeplitz or Hankel determinant, related to orthogonal polynomials on the unit circle. Indeed, this correspondence is more general.

For the unitary group:

Theorem 2.1. Let $f(z), g(z)$ be any functions on the unit circle. Then

$$
\mathrm{E}_{U \in U(l)} \operatorname{det}(f(U)) \operatorname{det}\left(g\left(U^{\dagger}\right)\right)=\operatorname{det}\left(\frac{1}{2 \pi} \int_{[0,2 \pi]} f\left(e^{i \theta}\right) g\left(e^{-i \theta}\right) e^{i(j-k) \theta} d \theta\right)_{0 \leq j, k<l} .
$$

Proof. Using the Weyl integration formula for the unitary group, we have

$$
\mathrm{E}_{U \in U(l)} \operatorname{det}(f(U)) \operatorname{det}\left(g\left(U^{\dagger}\right)\right)=\frac{1}{l !(2 \pi)^{l}} \int_{[0,2 \pi]^{l}} \prod_{0 \leq j<k<l}\left|e^{i j \theta}-e^{i k \theta}\right|^{2} \prod_{0 \leq j<l} f\left(e^{i \theta_{j}}\right) g\left(e^{-i \theta_{j}}\right) d \theta_{j} .
$$

The result follows from the standard theory of Toeplitz determinants, or by the classic formula for the integral of a product of two (generalized) Vandermonde determinants (see, for instance, 9]):

$$
\frac{1}{l !} \int_{S^{l}} \operatorname{det}\left(\phi_{j}\left(x_{k}\right)\right)_{0 \leq j, k<l} \operatorname{det}\left(\psi_{j}\left(x_{k}\right)\right)_{0 \leq j, k<l} \prod_{j} \mu\left(d x_{j}\right)=\operatorname{det}\left(\int_{S} \phi_{j}(x) \psi_{k}(x) \mu(d x)\right)_{0 \leq j, k<l},
$$

for any measure $\mu$ on any set $S$.

Similarly, for the orthogonal and symplectic groups, we have: 
Theorem 2.2. Let $g(z)$ be any function on the unit circle such that the integrals

$$
\iota_{j}=\frac{1}{2 \pi} \int_{[0,2 \pi]} g\left(e^{i \theta}\right) g\left(e^{-i \theta}\right) e^{i j \theta} d \theta
$$

are well-defined. Then

$$
\begin{aligned}
\mathrm{E}_{U \in O^{+}(2 l)} \operatorname{det}(g(U)) & =\frac{1}{2} \operatorname{det}\left(\iota_{j-k}+\iota_{j+k}\right)_{0 \leq j, k<l} \\
\mathrm{E}_{U \in O^{-}(2 l)} \operatorname{det}(g(U)) & =g(1) g(-1) \operatorname{det}\left(\iota_{j-k}-\iota_{j+k+2}\right)_{0 \leq j, k<l-1} \\
\mathrm{E}_{U \in O^{+}(2 l+1)} \operatorname{det}(g(U)) & =g(1) \operatorname{det}\left(\iota_{j-k}-\iota_{j+k+1}\right)_{0 \leq j, k<l} \\
\mathrm{E}_{U \in O^{-}(2 l+1)} \operatorname{det}(g(U)) & =g(-1) \operatorname{det}\left(\iota_{j-k}+\iota_{j+k+1}\right)_{0 \leq j, k<l} \\
\mathrm{E}_{U \in S p(2 l)} \operatorname{det}(g(U)) & =\operatorname{det}\left(\iota_{j-k}-\iota_{j+k+2}\right)_{0 \leq j, k<l},
\end{aligned}
$$

except that $\mathrm{E}_{U \in O^{+}(0)} \operatorname{det}(g(U))=1$.

Proof. As observed in Proposition 3.1 of [23], integrals over the orthogonal and symplectic groups can be expressed as Hankel determinants; thus, for instance,

$$
\mathrm{E}_{U \in O^{+}(2 l)} \operatorname{det}(g(U)) \propto \operatorname{det}\left(\frac{1}{\pi} \int_{[0,2 \pi]} g\left(e^{i \theta}\right) g\left(e^{-i \theta}\right) p_{j}(\cos (\theta)) p_{k}(\cos (\theta)) d \theta\right)_{0 \leq j, k<l}
$$

for any polynomials $p_{j}$ with $\operatorname{deg}\left(p_{j}\right)=j$. In particular, this must be true when $p_{j}(\cos (\theta))=\cos (j \theta)($ Chebyshev polynomials). In that case, noting that $\iota_{k}=\iota_{-k}$, the $j k$ coefficient of the determinant is

$$
\frac{1}{\pi} \int_{[0,2 \pi]} g\left(e^{i \theta}\right) g\left(e^{-i \theta}\right) \cos (j \theta) \cos (k \theta) d \theta=\iota_{j-k}+\iota_{j+k} .
$$

The constant of proportionality can be determined by comparing the two sides when $g=1$, and thus $\iota_{j}=\delta_{j 0}$.

For the other cases, we take $p_{j}(\cos (\theta))$ to be

$$
\frac{\sin ((j+1) \theta)}{\sin (\theta)}, \frac{\sin ((j+1 / 2) \theta)}{\sin (\theta / 2)}, \frac{\cos ((j+1 / 2) \theta)}{\cos (\theta / 2)}
$$

as appropriate.

For our purposes, we will need the following related result:

Theorem 2.3. Let $g(z)$ be as above, and consider the (symmetric) inner product on polynomials

$$
\langle p(z), q(z)\rangle=\frac{1}{2 \pi} \int_{[0,2 \pi]} p\left(e^{i \theta}\right) q\left(e^{-i \theta}\right) g\left(e^{i \theta}\right) g\left(e^{-i \theta}\right) d \theta .
$$

Let $\pi_{j}(z)$ be the monic orthogonal polynomials on the unit circle relative to that inner product, and define $N_{j}=\left\langle\pi_{j}(z), \pi_{j}(z)\right\rangle$. If the polynomials $\pi_{j}(z)$ are well-defined, then

$$
\begin{aligned}
\mathrm{E}_{U \in U(l)}|\operatorname{det}(g(U))|^{2} & =\prod_{0 \leq j<l} N_{j} \\
\mathrm{E}_{U \in O^{+}(0)} \operatorname{det}(g(U)) & =1
\end{aligned}
$$




$$
\begin{aligned}
\mathrm{E}_{U \in O^{+}(2 l)} \operatorname{det}(g(U)) & =N_{0} \prod_{0 \leq j<l-1} N_{2 j+2}\left(1+\pi_{2 j+2}(0)\right)^{-1} \\
\mathrm{E}_{U \in O^{-}(2 l)} \operatorname{det}(g(U)) & =g(1) g(-1) \prod_{0 \leq j<l-1} N_{2 j+2}\left(1-\pi_{2 j+2}(0)\right)^{-1} \\
\mathrm{E}_{U \in O^{+}(2 l+1)} \operatorname{det}(g(U)) & =g(1) \prod_{0 \leq j<l} N_{2 j+1}\left(1-\pi_{2 j+1}(0)\right)^{-1} \\
\mathrm{E}_{U \in O^{-}(2 l+1)} \operatorname{det}(g(U)) & =g(-1) \prod_{0 \leq j<l} N_{2 j+1}\left(1+\pi_{2 j+1}(0)\right)^{-1} \\
\mathrm{E}_{U \in S p(2 l)} \operatorname{det}(g(U)) & =\prod_{0 \leq j<l} N_{2 j+2}\left(1-\pi_{2 j+2}(0)\right)^{-1} .
\end{aligned}
$$

The proof will be given in Section 3 below. By combining these formulae, we obtain:

Corollary 2.4. Let $g(z)$ be as above. Then for any $l>0$,

$$
g(1) g(-1) \mathrm{E}_{U \in U(l)}|\operatorname{det}(g(U))|^{2}=\left(\mathrm{E}_{U \in O^{+}(l+1)} \operatorname{det}(g(U))\right)\left(\mathrm{E}_{U \in O^{-}(l+1)} \operatorname{det}(g(U))\right) .
$$

Proof. Use $N_{l}=\left(1-\pi_{l}(0)^{2}\right) N_{l-1}$ which follows from (3.11) below.

In our case, the function $g(z)=e^{t z}$, and thus everything is related to the orthogonal polynomials on the circle for the weight function $\exp (t(z+1 / z))=\exp (2 t \cos \theta)$. Let these polynomials be $\pi_{l}(z ; t)$ with norms $N_{l}(t)$, and define five functions $D_{l}(t), D^{ \pm \pm}(t)$ by

$$
\begin{aligned}
D_{l}(t) & =\operatorname{det}\left(I_{j-k}(2 t)\right)_{0 \leq j, k<l} \\
& =\prod_{0 \leq j<l} N_{j}(t) \\
D_{0}^{--}(t) & =1 \\
D_{l}^{--}(t) & =\frac{1}{2} \operatorname{det}\left(I_{j-k}(2 t)+I_{j+k}(2 t)\right)_{0 \leq j, k<l} \\
& =N_{0}(t) \prod_{0 \leq j<l-1} N_{2 j+2}(t)\left(1+\pi_{2 j+2}(0 ; t)\right)^{-1} \\
D_{l}^{++}(t) & =\operatorname{det}\left(I_{j-k}(2 t)-I_{j+k+2}(2 t)\right)_{0 \leq j, k<l} \\
& =\prod_{0 \leq j<l} N_{2 j+2}(t)\left(1-\pi_{2 j+2}(0 ; t)\right)^{-1} \\
D_{l}^{+-}(t) & =\operatorname{det}\left(I_{j-k}(2 t)-I_{j+k+1}(2 t)\right)_{0 \leq j, k<l} \\
& =\prod_{0 \leq j<l} N_{2 j+1}(t)\left(1-\pi_{2 j+1}(0 ; t)\right)^{-1} \\
D_{l}^{-+}(t) & =\operatorname{det}\left(I_{j-k}(2 t)+I_{j+k+1}(2 t)\right)_{0 \leq j, k<l} \\
& =\prod_{0 \leq j<l} N_{2 j+1}(t)\left(1+\pi_{2 j+1}(0 ; t)\right)^{-1},
\end{aligned}
$$

where

$$
I_{j}(2 t)=\frac{1}{2 \pi} \int_{[0,2 \pi]} e^{2 t \cos \theta} e^{i j \theta}=\sum_{m} \frac{t^{m}}{m !} \frac{t^{j+m}}{(j+m) !}
$$


is a modified Bessel function of the first kind.

Then

$$
\begin{aligned}
\mathrm{E}_{U \in U(l)}|\exp (t \operatorname{Tr}(U))|^{2} & =D_{l}(t) \\
\mathrm{E}_{U \in O^{+}(2 l)} \exp (t \operatorname{Tr}(U)) & =D_{l}^{--}(t) \\
\mathrm{E}_{U \in O^{-}(2 l)} \exp (t \operatorname{Tr}(U)) & =D_{l-1}^{++}(t) \\
\mathrm{E}_{U \in O^{+}(2 l+1)} \exp (t \operatorname{Tr}(U)) & =e^{t} D_{l}^{+-}(t) \\
\mathrm{E}_{U \in O^{-}(2 l+1)} \exp (t \operatorname{Tr}(U)) & =e^{-t} D_{l}^{-+}(t) .
\end{aligned}
$$

In summary:

Theorem 2.5. For $l \geq 0$, we have the following formulae:

$$
\begin{aligned}
P_{l}^{\square}(t) & =e^{-t^{2}} D_{l}(t) \\
P_{2 l}^{\square}(t) & =e^{-t^{2} / 2}\left[D_{l}^{--}(t)+D_{l-1}^{++}(t)\right] / 2 \\
P_{2 l+1}^{\square}(t) & =e^{-t^{2} / 2}\left[e^{t} D_{l}^{+-}(t)+e^{-t} D_{l}^{-+}(t)\right] / 2 \\
P_{2 l}^{\square}(t) & =e^{-t^{2} / 2} D_{l}^{++}(t), \\
P_{2 l}^{\square}(t) & =e^{-2 t^{2}} D_{l}(t)^{2} \\
P_{2 l+1}^{\square}(t) & =e^{-2 t^{2}} D_{l}(t) D_{l+1}(t) \\
P_{2 l}^{\bigotimes}(t) & =e^{-t^{2}} D_{l}(t)
\end{aligned}
$$

except that $P_{0}^{\square}(t)=e^{-t^{2} / 2} D_{0}^{--}(t)=e^{-t^{2} / 2}$.

In [2], we will also need the following limits, which follow immediately from Szegö's limit theorem and the analogue [23] for orthogonal polynomials on a finite interval:

Theorem 2.6. For any real $t \geq 0$,

$$
\begin{aligned}
\lim _{l \rightarrow \infty} D_{l}(t) & =e^{t^{2}} \\
\lim _{l \rightarrow \infty} D_{l}^{--}(t) & =e^{t^{2} / 2} \\
\lim _{l \rightarrow \infty} D_{l}^{++}(t) & =e^{t^{2} / 2} \\
\lim _{l \rightarrow \infty} D_{l}^{+-}(t) & =e^{-t+t^{2} / 2} \\
\lim _{l \rightarrow \infty} D_{l}^{-+}(t) & =e^{t+t^{2} / 2} .
\end{aligned}
$$

Remark. These limits are also valid as limits of formal power series in $t$; see [10] and Theorem 8.10 below. 
Corollary 2.7. For any real $t \geq 0$,

$$
\begin{aligned}
e^{-t^{2}} D_{l}(t) & =\prod_{j \geq l} N_{j}(t)^{-1} \\
e^{-t^{2} / 2} D_{l}^{--}(t) & =\prod_{j \geq l} N_{2 j+2}(t)^{-1}\left(1+\pi_{2 j+2}(0 ; t)\right) \\
e^{-t^{2} / 2} D_{l}^{++}(t) & =\prod_{j \geq l} N_{2 j+2}(t)^{-1}\left(1-\pi_{2 j+2}(0 ; t)\right) \\
e^{-t^{2} / 2+t} D_{l}^{+-}(t) & =\prod_{j \geq l} N_{2 j+1}(t)^{-1}\left(1-\pi_{2 j+1}(0 ; t)\right) \\
e^{-t^{2} / 2-t} D_{l}^{-+}(t) & =\prod_{j \geq l} N_{2 j+1}(t)^{-1}\left(1+\pi_{2 j+1}(0 ; t)\right) .
\end{aligned}
$$

\section{Orthogonal polynomial identities}

Let $w(x)$ on $[-1,1]$ and $f(z)$ on the unit circle be related by

$$
f\left(e^{i \theta}\right)=w(\cos \theta) .
$$

Associated to $f$ are five sets of monic orthogonal polynomials: $\pi_{l}(z)$ with respect to the (symmetric) inner product

$$
\langle p(z), q(z)\rangle=\frac{1}{2 \pi} \int_{[0,2 \pi]} p\left(e^{i \theta}\right) q\left(e^{-i \theta}\right) f\left(e^{i \theta}\right) d \theta .
$$

on the unit circle, and the four sets $p_{l}^{ \pm \pm}(z)$, with respect to the inner products

$$
\langle p(x), q(x)\rangle=\frac{1}{\pi} \int_{[-1,1]} p(x) q(x) w(x)(1-x)^{ \pm 1 / 2}(1+x)^{ \pm 1 / 2} d x .
$$

The notation $\langle p, q\rangle$ will refer to the inner product with respect to $f(z)$ or $w(x)\left(1-x^{2}\right)^{-1 / 2}$, whichever is appropriate. Thus the defining identities for these polynomials are:

$$
\begin{aligned}
\left\langle\pi_{n}, \pi_{m}\right\rangle & =\delta_{n m} N_{n} \\
\left\langle p_{n}^{--}, p_{m}^{--}\right\rangle & =\delta_{n m} N_{n}^{--} \\
\left\langle(1+x) p_{n}^{-+}, p_{m}^{-+}\right\rangle & =\delta_{n m} N_{n}^{-+} \\
\left\langle(1-x) p_{n}^{+-}, p_{m}^{+-}\right\rangle & =\delta_{n m} N_{n}^{+-} \\
\left\langle\left(1-x^{2}\right) p_{n}^{++}, p_{m}^{++}\right\rangle & =\delta_{n m} N_{n}^{++} .
\end{aligned}
$$

We also use the notation

$$
\pi_{l}^{*}(z)=z^{l} \pi_{l}(1 / z)
$$

Then the following identities hold (see for example Theorem 11.5 of [39]): For $\pi$ :

$$
\pi_{l+1}(z)=z \pi_{l}(z)+\pi_{l+1}(0) \pi_{l}^{*}(z)
$$




$$
\begin{aligned}
N_{l} & =N_{0} \prod_{1 \leq j \leq l}\left(1-\pi_{j}(0)^{2}\right) \\
\pi_{l}(1) & =\prod_{1 \leq j \leq l}\left(1+\pi_{j}(0)\right) \\
(-1)^{l} \pi_{l}(-1) & =\prod_{1 \leq j \leq l}\left(1+(-1)^{j} \pi_{j}(0)\right) .
\end{aligned}
$$

For $p^{--}$:

$$
\begin{aligned}
p_{0}^{--}((z+1 / z) / 2) & =1 \\
(2 z)^{l} p_{l}^{--}((z+1 / z) / 2) & =\pi_{2 l-1}^{*}(z)+z \pi_{2 l-1}(z) \\
p_{l}^{--}(1) & =2^{-l} \prod_{0 \leq j<2 l}\left(1+\pi_{j}(0)\right) \\
p_{l}^{--}(-1) & =(-2)^{-l} \prod_{0 \leq j<2 l}\left(1+(-1)^{j} \pi_{j}(0)\right) \\
N_{l}^{--} & =4^{1 / 2-l}\left(1+\pi_{2 l}(0)\right)^{-1} \prod_{0 \leq j<2 l}\left(1-\pi_{j}(0)^{2}\right) \\
& =4^{1 / 2-l} N_{2 l}\left(1+\pi_{2 l}(0)\right)^{-1}
\end{aligned}
$$

For $p^{+-}$:

$$
\begin{aligned}
(1-z)(2 z)^{l} p_{l}^{+-}((z+1 / z) / 2) & =\pi_{2 l}^{*}(z)-z \pi_{2 l}(z) \\
p_{l}^{+-}(-1) & =(-2)^{-l} \prod_{1 \leq j \leq 2 l}\left(1+(-1)^{j} \pi_{j}(0)\right) \\
N_{l}^{+-} & =4^{-l}\left(1+\pi_{2 l+1}(0)\right) \prod_{1 \leq j \leq 2 l}\left(1-\pi_{j}(0)^{2}\right) \\
& =4^{-l} N_{2 l}\left(1+\pi_{2 l+1}(0)\right) \\
& =4^{-l} N_{2 l+1}\left(1-\pi_{2 l+1}(0)\right)^{-1}
\end{aligned}
$$

For $p^{-+}$:

$$
\begin{aligned}
(1+z)(2 z)^{l} p_{l}^{-+}((z+1 / z) / 2) & =\pi_{2 l}^{*}(z)+z \pi_{2 l}(z) \\
p_{l}^{-+}(1) & =2^{-l} \prod_{1 \leq i \leq 2 l}\left(1+\pi_{j}(0)\right) \\
N_{l}^{-+} & =4^{-l}\left(1-\pi_{2 l+1}(0)\right) \prod_{1 \leq i \leq 2 l}\left(1-\pi_{i}(0)^{2}\right) \\
& =4^{-l} N_{2 l}\left(1-\pi_{2 l+1}(0)\right) \\
& =4^{-l} N_{2 l+1}\left(1+\pi_{2 l+1}(0)\right)^{-1}
\end{aligned}
$$


And finally, for $p^{++}$:

$$
\begin{aligned}
\left(1-z^{2}\right)(2 z)^{l} p_{l}^{++}((z+1 / z) / 2) & =\pi_{2 l+1}^{*}(z)-z \pi_{2 l+1}(z) \\
N_{l}^{++} & =4^{-l-1 / 2}\left(1+\pi_{2 l+2}(0)\right) \prod_{1 \leq j \leq 2 l+1}\left(1-\pi_{j}(0)^{2}\right) \\
& =4^{-l-1 / 2} N_{2 l+1}\left(1+\pi_{2 l+2}(0)\right) \\
& =4^{-l-1 / 2} N_{2 l+2}\left(1-\pi_{2 l+2}(0)\right)^{-1}
\end{aligned}
$$

All of the proofs are straightforward.

We can now prove Theorem 2.3. Recall that we have chosen $f$ to be of the form $f(z)=g(z) g(1 / z)$ such that the above inner product is well-defined and nondegenerate. Now consider the integral for $O^{+}(2 l)$. By the proof of Theorem 2.2 and the theory of Hankel determinants, we have

$$
\mathrm{E}_{U \in O^{+}(2 l)} \operatorname{det}(g(U)) \propto \prod_{0 \leq j<l} N_{j}^{++}
$$

but then this is in turn proportional to

$$
N_{0} \prod_{0 \leq j<l-1} N_{2 j+2}\left(1+\pi_{2 j+2}(0)\right)^{-1} .
$$

The constant can be determined by taking $g=1$. The other cases are analogous.

Theorem 3.1. Let $f$ and $g$ be as above. Then for any $\alpha$,

$$
\begin{aligned}
\mathrm{E}_{U \in O^{ \pm}(2 l)} \operatorname{det}((1-\alpha U) g(U)) & =\left(\pi_{2 l-1}^{*}(\alpha) \pm \alpha \pi_{2 l-1}(\alpha)\right) \mathrm{E}_{U \in O^{ \pm}(2 l)} \operatorname{det}(g(U)) \\
\mathrm{E}_{U \in O^{ \pm}(2 l+1)} \operatorname{det}((1-\alpha U) g(U)) & =\left(\pi_{2 l}^{*}(\alpha) \mp \alpha \pi_{2 l}(\alpha)\right) \mathrm{E}_{U \in O^{ \pm}(2 l+1)} \operatorname{det}(g(U))
\end{aligned}
$$

As a special case,

$$
\begin{aligned}
\mathrm{E}_{U \in O^{+}(l)} \operatorname{det}((1+U) g(U)) & =2 g(-1)^{-1} \mathrm{E}_{U \in O^{-}(l+1)} \operatorname{det}(g(U)) \\
\mathrm{E}_{U \in O^{-}(l)} \operatorname{det}((1+U) g(U)) & =0 \\
\mathrm{E}_{U \in O^{+}(2 l)} \operatorname{det}((1-U) g(U)) & =2 g(1)^{-1} \mathrm{E}_{U \in O^{+}(2 l+1)} \operatorname{det}(g(U)) \\
\mathrm{E}_{U \in O^{-}(2 l)} \operatorname{det}((1-U) g(U)) & =0 \\
\mathrm{E}_{U \in O^{+}(2 l+1)} \operatorname{det}((1-U) g(U)) & =0 \\
\mathrm{E}_{U \in O^{-}(2 l+1)} \operatorname{det}((1-U) g(U)) & =2 g(1)^{-1} \mathrm{E}_{U \in O^{-}(2 l+2)} \operatorname{det}(g(U))
\end{aligned}
$$

Proof. Follows by standard results about the behavior of orthogonal polynomials when the weight function is multiplied by a polynomial.

Similarly, 
Theorem 3.2. With notation as above, and for $\alpha \beta \neq 1$,

$$
\mathrm{E}_{U \in U(l)} \operatorname{det}\left((1-\alpha U)\left(1-\beta U^{\dagger}\right) g(U) g\left(U^{\dagger}\right)\right)=\frac{\pi_{l}^{*}(\alpha) \pi_{l}^{*}(\beta)-\alpha \beta \pi_{l}(\alpha) \pi_{l}(\beta)}{1-\alpha \beta} \mathrm{E}_{U \in U(l)} \operatorname{det}\left(g(U) g\left(U^{\dagger}\right)\right)
$$

In particular,

$$
\begin{aligned}
2 g(1) g(-1)(1-\alpha \beta) \mathrm{E}_{U \in U(l)} & \operatorname{det}\left((1-\alpha U)\left(1-\beta U^{\dagger}\right) g(U) g\left(U^{\dagger}\right)\right) \\
= & \mathrm{E}_{U \in O^{+}(l+1)} \operatorname{det}((1-\alpha U) g(U)) \mathrm{E}_{U \in O^{-}(l+1)} \operatorname{det}((1-\beta U) g(U)) \\
& +\mathrm{E}_{U \in O^{+}(l+1)} \operatorname{det}((1-\beta U) g(U)) \mathrm{E}_{U \in O^{-}(l+1)} \operatorname{det}((1-\alpha U) g(U))
\end{aligned}
$$

For our purposes, the following form is preferable:

Corollary 3.3. With notation as above, and for $\alpha \beta \neq 1,|\beta|<1$,

$$
\begin{aligned}
\frac{2 g(1) g(-1)(1-\alpha \beta)}{\left(1-\beta^{2}\right)} \mathrm{E}_{U \in U(l)} & \operatorname{det}\left((1-\alpha U)(1-\beta U)^{-1} g(U) g\left(U^{\dagger}\right)\right) \\
= & \mathrm{E}_{U \in O^{+}(l+1)} \operatorname{det}\left((1-\alpha U)(1-\beta U)^{-1} g(U)\right) \mathrm{E}_{U \in O^{-}(l+1)} \operatorname{det}(g(U)) \\
+ & \mathrm{E}_{U \in O^{+}(l+1)} \operatorname{det}(g(U)) \mathrm{E}_{U \in O^{-}(l+1)} \operatorname{det}\left((1-\alpha U)(1-\beta U)^{-1} g(U)\right)
\end{aligned}
$$

Remark. This, together with the following theorem, enables us to compute the limits $\alpha \rightarrow \pm 1$ or $\beta \rightarrow \pm 1$.

Theorem 3.4. With notation as above, and for $|\beta|<1$,

$$
\begin{aligned}
& \mathrm{E}_{U \in O^{+}(2 l+2)} \operatorname{det}\left((1-\beta U)^{-1} g(U)\right) / \mathrm{E}_{U \in O^{+}(2 l+2)} \operatorname{det}(g(U))=\frac{\frac{1}{2} \beta^{-l}\left\langle 2^{l} p_{l}^{--}(x),\left(1-\beta^{2}\right) /\left(1-2 \beta x+\beta^{2}\right)\right\rangle}{\left(1-\beta^{2}\right) N_{2 l}\left(1+\pi_{2 l}(0)\right)^{-1}} \\
& \mathrm{E}_{U \in O^{-}(2 l+2)} \operatorname{det}\left((1-\beta U)^{-1} g(U)\right) / \mathrm{E}_{U \in O^{-}(2 l+2)} \operatorname{det}(g(U))=\frac{\beta^{1-l}\left\langle 2^{l}\left(1-x^{2}\right) p_{l-1}^{++}(x), 1 /\left(1-2 \beta x+\beta^{2}\right)\right\rangle}{\left(1-\beta^{2}\right) N_{2 l}\left(1-\pi_{2 l}(0)\right)^{-1}} \\
& \mathrm{E}_{U \in O^{+}(2 l+3)} \operatorname{det}\left((1-\beta U)^{-1} g(U)\right) / \mathrm{E}_{U \in O^{+}(2 l+3)} \operatorname{det}(g(U))=\frac{\beta^{-l}\left\langle 2^{l}(1-x) p_{l}^{+-}(x),(1+\beta) /\left(1-2 \beta x+\beta^{2}\right)\right\rangle}{\left(1-\beta^{2}\right) N_{2 l}\left(1+\pi_{2 l+1}(0)\right)}
\end{aligned}
$$

$\mathrm{E}_{U \in O^{-}(2 l+3)} \operatorname{det}\left((1-\beta U)^{-1} g(U)\right) / \mathrm{E}_{U \in O^{-}(2 l+3)} \operatorname{det}(g(U))=\frac{\beta^{-l}\left\langle 2^{l}(1+x) p_{l}^{-+}(x),(1-\beta) /\left(1-2 \beta x+\beta^{2}\right)\right\rangle}{\left(1-\beta^{2}\right) N_{2 l}\left(1-\pi_{2 l+1}(0)\right)}$

$$
\mathrm{E}_{U \in S p(2 l)} \operatorname{det}\left((1-\beta U)^{-1} g(U)\right) / \mathrm{E}_{U \in S p(2 l)} \operatorname{det}(g(U))=\frac{\beta^{1-l}\left\langle 2^{l}\left(1-x^{2}\right) p_{l-1}^{++}(x), 1 /\left(1-2 \beta x+\beta^{2}\right)\right\rangle}{N_{2 l}\left(1-\pi_{2 l}(0)\right)^{-1}} .
$$

In the limits as $\beta \rightarrow \pm 1$,

$$
\begin{aligned}
& \lim _{\substack{\beta \rightarrow 1 \\
|\beta|<1}}\left(1-\beta^{2}\right) \mathrm{E}_{U \in O^{ \pm}(l+1)} \operatorname{det}\left((1-\beta U)^{-1} g(U)\right)=g(1) \mathrm{E}_{U \in O^{ \pm}(l)} \operatorname{det}(g(U)) \\
& \lim _{\substack{\beta \rightarrow-1 \\
|\beta|<1}}\left(1-\beta^{2}\right) \mathrm{E}_{U \in O^{ \pm}(l+1)} \operatorname{det}\left((1-\beta U)^{-1} g(U)\right)=g(-1) \mathrm{E}_{U \in O^{\mp}(l)} \operatorname{det}(g(U))
\end{aligned}
$$


For the symplectic group,

$$
\begin{aligned}
& \lim _{\substack{\beta \rightarrow 1 \\
|\beta|<1}} \mathrm{E}_{U \in S p(2 l)} \operatorname{det}\left((1-\beta U)^{-1} g(U)\right)=g(-1)^{-1} \mathrm{E}_{U \in O^{-}(2 l+1)} \operatorname{det}(g(U)) \\
& \lim _{\substack{\beta \rightarrow-1 \\
|\beta|<1}} \mathrm{E}_{U \in S p(2 l)} \operatorname{det}\left((1-\beta U)^{-1} g(U)\right)=g(1)^{-1} \mathrm{E}_{U \in O^{+}(2 l+1)} \operatorname{det}(g(U))
\end{aligned}
$$

Proof. The first group of statements are straightforward via appropriate row and column operations on the Hankel matrix.

For the limits, consider

$$
\lim _{\substack{\beta \rightarrow 1 \\|\beta|<1}}\left(1-\beta^{2}\right) \mathrm{E}_{U \in O^{+}(2 l+2)} \operatorname{det}(1-\beta U)^{-1} \operatorname{det}(g(U))
$$

Here we need to compute the limit of

$$
\beta^{-l}\left\langle 2^{l} p_{l}^{--}(x),\left(1-\beta^{2}\right) /\left(1-2 \beta x+\beta^{2}\right)\right\rangle .
$$

Now,

$$
\left(1-\beta^{2}\right) /\left(1-\beta(z+1 / z)+\beta^{2}\right)=1+\sum_{m>0} \beta^{m}\left(z^{m}+z^{-m}\right),
$$

so for any polynomial $q(z)$,

$$
\lim _{\substack{\beta \rightarrow 1 \\|\beta|<1}}\left\langle q(z), 1 /\left(1-2 \beta x+\beta^{2}\right)\right\rangle=q(1) g(1)^{2} .
$$

Plugging in and simplifying gives the desired result. The other cases are either analogous or follow from Theorem 3.1 above.

\section{Diagonal points}

Recall that in defining the ensembles $S^{\circledast}$ above, we insisted for $\square$ and $\nabla$ that $\pi$ have no fixed points, and for $\square$ and $\otimes$ that $\pi \iota$ have no fixed points. While these conditions are very natural from the standpoint of points in the square, they seem somewhat artificial in the permutation setting. This suggests consideration of the following extended ensembles:

$$
\begin{aligned}
& \tilde{S}_{n}^{\square}=\left\{\pi \in S_{n}: \pi=\pi^{-1}\right\} \\
& \tilde{S}_{n}^{\square}=\left\{\pi \in S_{n}: \pi=\iota \pi^{-1} \iota\right\} \\
& \tilde{S}_{n}^{\square}=\left\{\pi \in S_{2 n}: \pi=\pi^{-1}, \pi=\iota \pi^{-1} \iota\right\} .
\end{aligned}
$$

We immediately encounter a difficulty, however, with the Poisson generating function, since the probabilities involve division by the complicated sum

$$
\sum_{0 \leq m \leq\lfloor n / 2\rfloor}\left(\begin{array}{c}
n \\
2 m
\end{array}\right) \frac{(2 m) !}{2^{m} m !}
$$


for $\nabla$ and $\square$, and a similar sum for $\bowtie$. However, there is a generalized Poisson model for which the generating function is again tractable. For $\square$, the model is as follows: In a given infinitesimal time interval $[t, t+d t]$, we add two points (the images of a uniform random point) with probability (1/2)tdt and add one point (a point uniformly chosen on the $/$ diagonal) with probability $\alpha d t$. Then the probability that we have $n$ points at time $t$ is

$$
e^{-\alpha t-t^{2} / 2}\left(\sum_{0 \leq m \leq\lfloor n / 2\rfloor} \alpha^{n-2 m}\left(\begin{array}{c}
n \\
2 m
\end{array}\right) \frac{(2 m) !}{2^{m} m !}\right) t^{n} / n ! .
$$

Thus if we define $P_{l}^{\square}(t ; \alpha)$ to be the probability that the increasing subsequence length at time $t$ is at most $l$, then

$$
P_{l}^{\square}(t ; \alpha)=e^{-\alpha t-t^{2} / 2} \sum_{0 \leq n} \frac{t^{n}}{n !} \sum_{0 \leq m} \alpha^{m} \tilde{f}_{n m l}^{\square},
$$

where $\tilde{f}_{n m l}^{\square}$ is the number of involutions on $n$ elements with $m$ fixed points and no increasing subsequence of length greater than $l$. This is, of course, compatible with our previous notation, with $P_{l}^{\square}(t ; 0)=P_{l}^{\square}(t)$. Similarly, for $\square$, if we add negated points with probability $\beta d t$, we obtain the Poisson generating function

$$
P_{l}^{\square}(t ; \beta)=e^{-\beta t-t^{2} / 2} \sum_{0 \leq n} \frac{t^{n}}{n !} \sum_{0 \leq m} \beta^{m} \tilde{f}_{n m l}^{\square} .
$$

Finally, for $\otimes$, we have two parameters in the model; we add fixed points with probability $\alpha d t$, negated points with probability $\beta d t$, and generic points with probability $2 t d t$, obtaining

$$
P_{l}^{\bigotimes}(t ; \alpha, \beta)=e^{-\alpha t-\beta t-t^{2}} \sum_{0 \leq n} \frac{t^{n}}{n !} \sum_{0 \leq m_{+}, m_{-}} \alpha^{m_{+}} \beta^{m_{-}} \tilde{f}_{n m_{+} m_{-} l}^{\bigotimes} .
$$

The $\beta$ parameter is largely irrelevant, since:

Lemma 4.1. For all $l \geq 0, \alpha \geq 0$ and $\beta \geq 0$,

$$
\begin{aligned}
P_{2 l+1}^{\square}(t ; \beta) & =P_{2 l}^{\square}(t ; 0) \\
P_{2 l+1}^{\bigotimes}(t ; \alpha, \beta) & =P_{2 l}^{\bigotimes}(t ; \alpha, 0)
\end{aligned}
$$

Proof. The key observation is that if an increasing subsequence contains a given point off the \ diagonal, it can always be extended to contain the reflection of that point through that diagonal; moreover, no increasing subsequence can contain more than one point on that diagonal. Thus the point collection at time $t$ has increasing subset size at most $2 l+1$ if and only if the off-diagonal subset has increasing subset size at most $2 l$. Since the points were added via a generalized Poisson process, the off-diagonal subset itself corresponds to a generalized Poisson process; the lemma is then immediate.

We can again express these generating functions as integrals: 
Theorem 4.2. For all $l$,

$$
\begin{aligned}
P_{l}^{\square}(t ; \alpha) & =e^{-\alpha t-t^{2} / 2} \mathrm{E}_{U \in O(l)} \operatorname{det}(1+\alpha U) \exp (t \operatorname{Tr}(U)) \\
P_{l}^{\square}(t ; 1) & =e^{-t^{2} / 2} \mathrm{E}_{U \in O^{-}(l+1)} \exp (t \operatorname{Tr}(U)) \\
P_{2 l}^{\triangleright}(t ; \beta) & =e^{-\beta t-t^{2} / 2} \mathrm{E}_{U \in S p(2 l)} \operatorname{det}(1-\beta U)^{-1} \exp (t \operatorname{Tr}(U)) \\
P_{2 l+1}^{\square}(t ; \beta) & =e^{-t^{2} / 2} \mathrm{E}_{U \in S p(2 l)} \exp (t \operatorname{Tr}(U)) \\
P_{l}^{\square}(t ; 1) & =e^{-t^{2} / 2} \mathrm{E}_{U \in O^{-}(l+1)} \exp (t \operatorname{Tr}(U)) \\
P_{2 l}^{\bigotimes}(t ; \alpha, \beta) & =e^{-\alpha t-\beta t-t^{2}} \mathrm{E}_{U \in U(l)} \operatorname{det}\left((1+\alpha U)(1-\beta U)^{-1}\right) \exp (2 t \operatorname{Re} \operatorname{Tr}(U)) \\
P_{2 l+1}^{\bigotimes}(t ; \alpha, \beta) & =e^{-\alpha t-t^{2}} \mathrm{E}_{U \in U(l)} \operatorname{det}(1+\alpha U) \exp (2 t \operatorname{Re} \operatorname{Tr}(U)) \\
P_{2 l+1}^{\bigotimes}(t ; 1, \beta) & =e^{-t^{2}} \mathrm{E}_{U \in O^{-}(l+2)} \exp (t \operatorname{Tr}(U)) \cdot \mathrm{E}_{U \in O^{-}(l+1)} \exp (t \operatorname{Tr}(U)) .
\end{aligned}
$$

Proof. This follows immediately from the symmetric function identities of Section 5 below. (See, in particular, remark 2 to Corollary 5.5.)

Remark. Strictly speaking, equations (4.13) and (4.16) are only valid when $\beta<1$. The correct formulae for $\beta \geq 1$ are obtained by analytic continuation (e.g., by expanding $\operatorname{det}(1-\beta U)^{-1}$ in a formal power series, integrating term-by-term, and summing).

From Theorem 4.2 and the orthogonal polynomial identities of Section 3 below, we have the following formulae.

Corollary 4.3. For $\alpha, \beta \geq 0$,

$$
\begin{aligned}
P_{2 l}^{\square}(t ; \alpha) & =e^{-\alpha t-t^{2} / 2} \frac{1}{2}\left\{\left[\pi_{2 l-1}^{*}(-\alpha ; t)-\alpha \pi_{2 l-1}(-\alpha ; t)\right] D_{l}^{--}(t)+\left[\pi_{2 l-1}^{*}(-\alpha ; t)+\alpha \pi_{2 l-1}(-\alpha ; t)\right] D_{l-1}^{++}(t)\right\}, \\
P_{2 l+1}^{\square}(t ; \alpha) & =e^{-\alpha t-t^{2} / 2} \frac{1}{2}\left\{\left[\pi_{2 l}^{*}(-\alpha ; t)+\alpha \pi_{2 l}(-\alpha ; t)\right] e^{t} D_{l}^{+-}(t)+\left[\pi_{2 l}^{*}(-\alpha ; t)-\alpha \pi_{2 l}(-\alpha ; t)\right] e^{-t} D_{l}^{-+}(t)\right\}, \\
P_{2 l}^{\square}(t ; 1) & =P_{2 l}^{\square}(t ; 1)=e^{-t-t^{2} / 2} D_{l}^{-+}(t), \\
P_{2 l+1}^{\square}(t ; 1) & =P_{2 l+1}^{\square}(t ; 1)=e^{-t^{2} / 2} D_{l}^{++}(t), \\
P_{2 l+1}^{\square}(t ; \beta) & =e^{-t^{2} / 2} D_{l}^{++}(t), \\
P_{2 l+1}^{\bigotimes}(t ; \alpha, \beta) & =e^{-\alpha t-t^{2}} \pi_{l}^{*}(-\alpha ; t) D_{l}(t), \\
P_{4 l+1}^{\bigotimes}(t ; 1, \beta) & =e^{-t-t^{2}} D_{l}^{++}(t) D_{l}^{-+}(t), \\
P_{4 l+3}^{\bigotimes}(t ; 1, \beta) & =e^{-t-t^{2}} D_{l}^{++}(t) D_{l+1}^{-+}(t) .
\end{aligned}
$$

\section{Schur function identities}

In order to derive the above generating functions for $P^{\square}(t ; \alpha), P^{\square}(t ; \beta)$ and $P^{\bigotimes}(t ; \alpha, \beta)$, we will first need a stronger version of Theorem 1.2. 
Remark. In the sequel, we will see a number of expressions of the form

$$
\mathrm{E}_{U \in U \circledast(l)} \operatorname{det}(g(U)),
$$

where $g$ takes values in a ring of formal power series (e.g., the ring of symmetric functions) with coefficients in $\mathbb{C}[z, 1 / z]$ (Laurent polynomials in $z$ ). This formal integral is defined by expanding $\operatorname{det}(g(U))$ and integrating term by term. We can recover analytical results by specializing down to a small number of variables in such a way that the resulting series converge.

We refer the reader to 29] for an introduction to symmetric functions. Recall the following symmetric function identities:

Lemma 5.1. The following identities hold in the algebra of symmetric functions on two sets of variables:

$$
\begin{aligned}
\prod_{j, k}\left(1-x_{j} y_{k}\right)^{-1} & =\sum_{\lambda} s_{\lambda}(x) s_{\lambda}(y) \\
& =\exp \left(\sum_{m>0} \frac{1}{m} p_{m}(x) p_{m}(y)\right) .
\end{aligned}
$$

And for any symmetric function $f$,

$$
\left\langle f(x), \prod_{j, k}\left(1-x_{j} y_{k}\right)^{-1}\right\rangle=f(y) .
$$

It will be convenient to write

$$
\prod_{j, k}\left(1-x_{j} y_{k}\right)^{-1}=\prod_{j} H\left(x_{j} ; y\right),
$$

where

$$
H(u ; y):=\prod_{j}\left(1-u y_{j}\right)^{-1}=\sum_{j} h_{j} u^{j}
$$

is the generating function for the complete symmetric functions $h_{j}$; by convention, $h_{j}=0$ when $j<0$. In the sequel, we will also need the generating function

$$
E(u ; y):=\prod_{j}\left(1+u y_{j}\right)=\sum_{j} e_{j} u^{j}
$$

for the elementary symmetric functions.

Theorem 5.2. For all $l \geq 0$,

$$
\begin{gathered}
\sum_{\ell(\lambda) \leq l} s_{\lambda}(x) s_{\lambda}(y)=\mathrm{E}_{U \in U(l)} \operatorname{det}\left(H(U ; x) H\left(U^{\dagger} ; y\right)\right) \\
\sum_{\ell(\lambda) \leq l} s_{2 \lambda}(x)=\mathrm{E}_{U \in O(l)} \operatorname{det}(H(U ; x)) \\
\sum_{\ell(\lambda) \leq 2 l} s_{\lambda^{2}}(x)=\mathrm{E}_{U \in S p(2 l)} \operatorname{det}(H(U ; x))
\end{gathered}
$$


Proof. Consider the first identity. We have

$$
\mathrm{E}_{U \in U(l)} \operatorname{det}\left(H(U ; x) H\left(U^{\dagger} ; y\right)\right)=\sum_{\lambda, \mu} s_{\lambda}(x) s_{\mu}(y) \mathrm{E}_{U \in U(l)} s_{\lambda}(U) s_{\mu}\left(U^{\dagger}\right) .
$$

But the integral on the right is 1 when $\lambda=\mu$ and $\ell(\lambda), \lambda(\mu) \leq l$, and is 0 otherwise. The result follows immediately.

Similarly, the identity for $O(l)$ follows from the fact that

$$
\mathrm{E}_{U \in O(l)} s_{\lambda}(U)
$$

is 1 when $\lambda$ is even and $\ell(\lambda) \leq l$, and is 0 otherwise; and the identity for $S p(2 l)$ follows from the fact that

$$
\mathrm{E}_{U \in S p(2 l)} s_{\lambda}(U)
$$

is 1 when $\lambda^{\prime}$ is even and $\ell(\lambda) \leq l$ and is 0 otherwise.

Remark 1. This is a direct generalization of the argument in [33].

Remark 2. The expression $\operatorname{det}(H(U ; x))$ can also be written as

$$
\exp \left(\sum_{m>0} \frac{1}{m} p_{m}(x) \operatorname{Tr}\left(U^{m}\right)\right) .
$$

For the hyperoctahedral cases $\square$ and $\bowtie$, we will need the notation

$$
\tilde{s}_{\lambda}(x)=s_{\lambda(0)}(x) s_{\lambda(1)}(x)
$$

whenever $\lambda$ is a partition with trivial 2-core and 2-quotient $\left(\lambda^{(0)}, \lambda^{(1)}\right)$, and $\tilde{s}_{\lambda}(x)=0$ otherwise. From Ex. 1.5.24 of [29], this can also be written as

$$
\tilde{s}_{\lambda}(x)=(-1)^{f(\lambda) / 2} \phi_{2}\left(s_{\lambda}(x)\right),
$$

where $f(\lambda)$ is the number of odd parts of $\lambda$ (note that this is even when $\lambda$ has trivial 2-core), and $\phi_{2}$ is the homomorphism such that

$$
\begin{aligned}
\phi_{2}\left(h_{2 n}\right) & =h_{n} \\
\phi_{2}\left(h_{2 n+1}\right) & =0,
\end{aligned}
$$

or equivalently,

$$
\phi_{2}(H(u ; x))=H\left(u^{2} ; x\right) .
$$

Corollary 5.3. For all $l \geq 0$,

$$
\begin{gathered}
\sum_{\ell(\lambda) \leq 2 l} \tilde{s}_{\lambda}(x) \tilde{s}_{\lambda}(y)=\mathrm{E}_{U \in U^{\varpi}(2 l)} \operatorname{det}\left(H(U ; x) H\left(U^{\dagger} ; y\right)\right) \\
\sum_{\ell(\lambda) \leq l} \tilde{s}_{2 \lambda^{2}}(x)=\mathrm{E}_{U \in U^{\boxplus}(2 l)} \operatorname{det}(H(U ; x))
\end{gathered}
$$


Proof. This follows from the computations

$$
\begin{aligned}
\sum_{\ell(\lambda) \leq 2 l} \tilde{s}_{\lambda}(x) \tilde{s}_{\lambda}(y) & =\sum_{\ell(\lambda) \leq l, \ell(\mu) \leq l} s_{\lambda}(x) s_{\mu}(x) s_{\lambda}(y) s_{\mu}(y) \\
& =\left(\sum_{\ell(\lambda) \leq l} s_{\lambda}(x) s_{\lambda}(y)\right)^{2} \\
& =\left(\mathrm{E}_{U \in U(l)} \operatorname{det}\left(H(U ; x) H\left(U^{\dagger} ; y\right)\right)\right)^{2} \\
& =\mathrm{E}_{U_{1}, U_{2} \in U(l)} \operatorname{det}\left(H\left(U_{1} ; x\right) H\left(U_{1}^{\dagger} ; y\right)\right) \operatorname{det}\left(H\left(U_{2} ; x\right) H\left(U_{2}^{\dagger} ; y\right)\right) \\
& =\mathrm{E}_{U \in U(l) \oplus U(l)} \operatorname{det}\left(H(U ; x) H\left(U^{\dagger} ; y\right)\right)
\end{aligned}
$$

and

$$
\begin{aligned}
\sum_{\ell(\lambda) \leq l} \tilde{s}_{2 \lambda^{2}}(x) & =\sum_{\ell(\lambda) \leq l} s_{\lambda}(x) s_{\lambda}(x) \\
& =\mathrm{E}_{U \in U(l)} \operatorname{det}(H(U ; x)) \operatorname{det}\left(H\left(U^{\dagger} ; x\right)\right) \\
& =\mathrm{E}_{U \in U^{\bigotimes}(2 l)} \operatorname{det}(H(U ; x)) .
\end{aligned}
$$

The point of writing the integrands in the form $\operatorname{det}(H(U ; x))$ is that we know how to convert such integrals into determinants:

Theorem 5.4. For all $l \geq 0$,

$$
\begin{aligned}
\mathrm{E}_{U \in U(l)} \operatorname{det}\left(H(U ; x) H\left(U^{\dagger} ; y\right)\right) & =\operatorname{det}\left(g_{j-k}(x ; y)\right)_{0 \leq j, k<l} \\
\mathrm{E}_{U \in O^{+}(2 l)} \operatorname{det}(H(U ; x)) & =\frac{1}{2} \operatorname{det}\left(i_{j-k}(x)+i_{j+k}(x)\right)_{0 \leq j, k<l} \\
\mathrm{E}_{U \in O^{-}(2 l)} \operatorname{det}(H(U ; x)) & =H(1 ; x) H(-1 ; x) \operatorname{det}\left(i_{j-k}(x)-i_{j+k+2}(x)\right)_{0 \leq j, k<l-1} \\
\mathrm{E}_{U \in O^{+}(2 l+1)} \operatorname{det}(H(U ; x)) & =H(1 ; x) \operatorname{det}\left(i_{j-k}(x)-i_{j+k+1}(x)\right)_{0 \leq j, k<l} \\
\mathrm{E}_{U \in O^{-}(2 l+1)} \operatorname{det}(H(U ; x)) & =H(-1 ; x) \operatorname{det}\left(i_{j-k}(x)+i_{j+k+1}(x)\right)_{0 \leq j, k<l} \\
\mathrm{E}_{U \in S p(2 l)} \operatorname{det}(H(U ; x)) & =\operatorname{det}\left(i_{j-k}(x)-i_{j+k+2}(x)\right)_{0 \leq j, k<l},
\end{aligned}
$$

where we define

$$
\begin{aligned}
g_{j}(x ; y) & =\sum_{m} h_{m}(x) h_{m+j}(y) \\
i_{j}(x) & =\sum_{m} h_{m}(x) h_{m+j}(x) .
\end{aligned}
$$

Proof. By Theorems 2.1 and 2.2, it suffices to evalute

$$
\frac{1}{2 \pi} \int_{[0,2 \pi]} H\left(e^{i \theta} ; x\right) H\left(e^{-i \theta} ; y\right) e^{i j \theta} d \theta .
$$


But this integral is just the coefficient of $z^{-j}$ in $H(z ; x) H\left(z^{-1} ; y\right)$; that is,

$$
\sum_{m} h_{m}(x) h_{m+j}(y)=g_{j}(x ; y) .
$$

The following is then immediate:

Corollary 5.5. For all $l>0$,

$$
\begin{aligned}
\sum_{\ell(\lambda) \leq l} s_{\lambda}(x) s_{\lambda}(y) & =\operatorname{det}\left(g_{j-k}(x ; y)\right)_{0 \leq j, k<l} \\
\sum_{\ell(\lambda) \leq 2 l} s_{2 \lambda}(x) & =\left[\frac{1}{2} \operatorname{det}\left(i_{j-k}(x)+i_{j+k}(x)\right)_{0 \leq j, k<l}+H(1 ; x) H(-1 ; x) \operatorname{det}\left(i_{j-k}(x)-i_{j+k+2}(x)\right)_{0 \leq j, k<l-1}\right] / 2 \\
\sum_{\ell(\lambda) \leq 2 l+1} s_{2 \lambda}(x) & =\left[H(1 ; x) \operatorname{det}\left(i_{j-k}(x)-i_{j+k+1}(x)\right)_{0 \leq j, k<l}+H(-1 ; x) \operatorname{det}\left(i_{j-k}(x)+i_{j+k+1}(x)\right)_{0 \leq j, k<l}\right] / 2 \\
\sum_{\ell(\lambda) \leq 2 l} s_{\lambda^{2}}(x) & =\operatorname{det}\left(i_{j-k}(x)-i_{j+k+2}(x)\right)_{0 \leq j, k<l}
\end{aligned}
$$

Remark 1. The expressions for $\sum_{\ell(\lambda) \leq l} s_{\lambda}(x) s_{\lambda}(y)$ and $\sum_{\ell(\lambda) \leq 2 l} s_{\lambda^{2}}(x)$ are already known (13] and [16], respectively; the latter also gives analogues of (5.64) and (5.65) below), although it is worth noting that the proof of the latter formula given here, in contrast to most of the proofs in the literature, involves neither pfaffians nor symplectic tableaux. The particular forms of (5.41) and (5.42) given here are new, although (C. Krattenthaler, personal communication) they can be readily derived from Theorem 2.4(3) of 31] together with well-known character formulas for symplectic and special orthogonal characters; see Remark 3 below. The analogue of (5.63), again given in terms of orthogonal and symplectic characters, is given in Theorem 2 of [27].

Remark 2. We recover our earlier identities by noting that when $|\lambda|=n,\left\langle p_{1^{n}}, s_{\lambda}(x)\right\rangle$ is equal to the number of tableaux of shape $\lambda$. So the generating functions for $f^{\circledast}$ are obtained by taking inner products of the above formulae with $\exp \left(t p_{1}(x)\right)$. But in general taking an inner product with the function

$$
\exp \left(\sum_{m>0} \frac{f_{m}}{m} p_{m}(x)\right)
$$

gives a homomorphism from the algebra of symmetric functions to $\mathbb{Q}\left[f_{1}, f_{2}, \ldots\right]$, given by the substitutions $p_{m}(x) \mapsto f_{m}$.

In our case, we have:

$$
\begin{aligned}
h_{m}(x) & \mapsto \frac{t^{m}}{m !} \\
H(u ; x) & \mapsto e^{u t} \\
i_{j}(x) & \mapsto I_{j}(2 t)
\end{aligned}
$$


so, for instance, we obtain

$$
\begin{aligned}
\sum_{n \geq 0} f_{n l} \frac{t^{2 n}}{n !^{2}} & =\mathrm{E}_{U \in U(l)} \exp (2 t \operatorname{Re} \operatorname{Tr}(U)) \\
& =\operatorname{det}\left(I_{j-k}(2 t)\right)_{0 \leq j, k<l},
\end{aligned}
$$

as before.

Remark 3. In [38], it is observed that the determinant

$$
\operatorname{det}\left(i_{j-k}(x)+i_{j-k+2}(x)\right)_{0 \leq j, k<l}
$$

is related by duality to a certain irreducible character of the symplectic group, and similarly the determinants of Corollary 5.7 below are related to irreducible characters of the (odd-dimensional) special orthogonal group, as are those of equations (5.41) and (5.42). It is not clear how this relates to our integrals, since the integrals are over groups of fixed dimension, while the characters are associated to groups of arbitrarily high dimension.

Remark 4. The approach in [16] for deriving such formulae is based on the operator

$$
f(z) \mapsto \phi\left(\prod_{1 \leq j<k \leq l}\left(1-z_{j}^{-1} z_{k}\right) f(z)\right)
$$

where $\phi$ is the linear operator on Laurent series taking $\prod_{1 \leq j \leq l} z_{j}^{b_{j}}$ to $\prod_{1 \leq j \leq l} h_{b_{j}}(x)$. Now, this operator can be expressed as an integral:

$$
\phi\left(\prod_{1 \leq j<k \leq l}\left(1-z_{j}^{-1} z_{k}\right) f(z)\right)=\int_{[0,2 \pi]^{l}} f\left(e^{i \theta}\right) \prod_{1 \leq j \leq l} H\left(e^{-i \theta_{j}} ; x\right) \prod_{1 \leq j<k \leq l}\left(1-e^{-i \theta_{j}} e^{i \theta_{k}}\right) \prod_{1 \leq j \leq l} d \theta_{j},
$$

which after symmetrizing the integrand becomes simply

$$
\mathrm{E}_{U \in U(l)} f(U) \operatorname{det}\left(H\left(U^{\dagger} ; x\right)\right)
$$

In [16], formulae are given for a generalization of $\sum_{\ell(\lambda) \leq l} s_{\lambda^{2}}(x)$, in which the sum is over all $\lambda$ with a given number of odd parts in the dual. This is derived using the homomorphism $H^{\perp}(\beta ; x)$ (29]) which takes $f(x)$ to $f(\beta, x)$ for any symmetric function $f$. The relevant property of $H^{\perp}(\beta ; x)$ is that

$$
H^{\perp}(\beta ; x) s_{\lambda}(x)=s_{\lambda}(\beta, x)=\sum_{\mu \lesssim \lambda} \beta^{|\lambda|-|\mu|} s_{\mu}(x),
$$

where $\mu \lesssim \lambda$ indicates that $\lambda-\mu$ is a "horizontal strip"; that is,

$$
\lambda_{i}^{\prime} \leq \mu_{i}^{\prime} \leq \lambda_{i}^{\prime}+1
$$

for all $i$, or equivalently

$$
\lambda_{1} \geq \mu_{1} \geq \lambda_{2} \geq \mu_{2} \geq \ldots
$$


We will also need the dual operator $E^{\perp}(\alpha ; x)$, which satisfies

$$
E^{\perp}(\alpha ; x) s_{\lambda}(x)=\sum_{\mu \lesssim^{\prime} \lambda} \alpha^{|\lambda|-|\mu|} s_{\mu}(x) .
$$

where

$$
\mu \lesssim \lambda \Leftrightarrow \mu^{\prime} \lesssim \lambda^{\prime}
$$

We have the identities

$$
\begin{aligned}
& H^{\perp}(\beta ; x) H(u ; x)=(1-\beta u)^{-1} H(u ; x) \\
& H^{\perp}(\beta ; x) E(u ; x)=(1+\beta u) E(u ; x) \\
& E^{\perp}(\alpha ; x) H(u ; x)=(1+\alpha u) H(u ; x) \\
& E^{\perp}(\alpha ; x) E(u ; x)=(1-\alpha u)^{-1} E(u ; x) .
\end{aligned}
$$

Theorem 5.6. For all $l>0$, we have the integral identities

$$
\begin{aligned}
\sum_{\ell(\lambda) \leq l} \alpha^{f(\lambda)} s_{\lambda}(x) & =\mathrm{E}_{U \in O(l)} \operatorname{det}((1+\alpha U) H(U ; x)) \\
\sum_{\ell(\lambda) \leq 2 l} \beta^{f\left(\lambda^{\prime}\right)} s_{\lambda}(x) & =\mathrm{E}_{U \in S p(2 l)} \operatorname{det}\left((1-\beta U)^{-1} H(U ; x)\right) \\
\sum_{\ell(\lambda) \leq 2 l+1} \beta^{f\left(\lambda^{\prime}\right)} s_{\lambda}(x) & =H(\beta ; x) \mathrm{E}_{U \in S p(2 l)} \operatorname{det}(H(U ; x))
\end{aligned}
$$

Proof. The point is that given any partition $\lambda$, there is a unique partition $\mu$ such that $\lambda \lesssim^{\prime} 2 \mu$; simply add one to each odd part of $\lambda$, then divide by 2 . In particular, then, $f(\lambda)=|2 \mu|-|\lambda|$, and thus

$$
\begin{aligned}
\sum_{\ell(\lambda) \leq l} \alpha^{f(\lambda)} s_{\lambda}(x) & =\sum_{\ell(\mu) \leq l} \sum_{\lambda \leq^{\prime} 2 \mu} \alpha^{|2 \mu|-|\lambda|} s_{\lambda}(x) \\
& =E^{\perp}(\alpha ; x) \sum_{\ell(\mu) \leq l} s_{2 \mu}(x) \\
& =\mathrm{E}_{U \in O(l)} E^{\perp}(\alpha ; x) \operatorname{det}(H(U ; x)) \\
& =\mathrm{E}_{U \in O(l)} \operatorname{det}((1+\alpha U) H(U ; x)) .
\end{aligned}
$$

Similarly,

$$
\begin{aligned}
\sum_{\ell(\lambda) \leq 2 l} \beta^{f\left(\lambda^{\prime}\right)} s_{\lambda}(x) & =H^{\perp}(\beta ; x) \mathrm{E}_{U \in S p(2 l)} \operatorname{det}(H(U ; x)) \\
& =\mathrm{E}_{U \in S p(2 l)} \operatorname{det}\left((1-\beta U)^{-1} H(U ; x)\right) .
\end{aligned}
$$

By the argument in [16], the expression for

$$
\sum_{\ell(\lambda) \leq 2 l+1} \beta^{f\left(\lambda^{\prime}\right)} s_{\lambda}(x)
$$


follows from Pieri's formula

$$
H(\beta ; x) s_{\mu}(x)=\sum_{\lambda \gtrsim \mu} \beta^{|\lambda|-|\mu|} s_{\lambda}(x)
$$

(eq. (5.16) in Chapter I of [29]), and the fact that for every $\lambda$ with $\ell(\lambda) \leq 2 l+1$, there is a unique $\mu$ with $\mu^{2} \lesssim \lambda$, and for that $\mu, \ell(\mu) \leq l$.

Remark. It is worth stressing here that the integral

$$
\mathrm{E}_{U \in S p(2 l)} \operatorname{det}\left((1-\beta U)^{-1} H(U ; x)\right)
$$

is a formal integral, in which the expression $(1-\beta z)^{-1}$ stands for the formal power series $\sum_{0 \leq m} z^{m} \beta^{m}$. We must therefore take special care when specializing to an analytical integral with $|\beta| \geq 1$.

Corollary 5.7. For all $l>0$,

$$
\sum_{\ell(\lambda) \leq l} s_{\lambda}(x)=E(1 ; x) \mathrm{E}_{U \in O^{-}(l+1)} \operatorname{det}(H(U ; x))
$$

so

$$
\begin{aligned}
\sum_{\ell(\lambda) \leq 2 l} s_{\lambda}(x) & =\operatorname{det}\left(i_{j-k}(x)+i_{j+k+1}(x)\right)_{0 \leq j, k<l} \\
\sum_{\ell(\lambda) \leq 2 l+1} s_{\lambda}(x) & =H(1 ; x) \operatorname{det}\left(i_{j-k}(x)-i_{j+k+2}(x)\right)_{0 \leq j, k<l}
\end{aligned}
$$

Proof. We have

$$
\begin{aligned}
\sum_{\ell(\lambda) \leq l} s_{\lambda}(x) & =\sum_{\ell(\lambda) \leq l} 1^{f(\lambda)} s_{\lambda}(x) \\
& =\mathrm{E}_{U \in O(l)} \operatorname{det}((1+U) H(U ; x)) \\
& =\frac{1}{2} \mathrm{E}_{U \in O^{+}(l)} \operatorname{det}((1+U) H(U ; x))
\end{aligned}
$$

since $\operatorname{det}(1+U)$ vanishes on $O^{-}(l)$. But then we can apply Theorem 3.1 to obtain

$$
\frac{1}{2} \mathrm{E}_{U \in O^{+}(l)} \operatorname{det}((1+U) H(U ; x))=H(-1 ; x)^{-1} \mathrm{E}_{U \in O^{-}(l+1)} \operatorname{det}(H(U ; x))
$$

as desired (recall $H(-t ; x) E(t ; x)=1)$. The remaining formulas are immediate.

Remark 1. Again, the determinantal forms are known [14] (based on an earlier pfaffian form 15]).

Remark 2. We could also have derived the formulae by the (formal) substitution $\beta=1$ in the above symplectic formulae, or by taking the limit $\beta \rightarrow 1^{-}$.

We also have the following formulae: 
Theorem 5.8. For all $l \geq 0$,

$$
\begin{gathered}
\sum_{\lambda_{2}^{\prime} \leq l} \alpha^{f(\lambda)} s_{\lambda}(x)=E(\alpha ; x) E_{U \in O(l)} \operatorname{det}(H(U ; x)) \\
\sum_{\lambda_{2}^{\prime} \leq l \leq \lambda_{1}^{\prime}} \alpha^{2 \lambda_{1}^{\prime}-l-f(\lambda)} s_{\lambda}(x)=E(\alpha ; x) E_{U \in O(l)} \operatorname{det}(U) \operatorname{det}(H(U ; x)) .
\end{gathered}
$$

Proof. We recall

$$
E(\alpha ; x) s_{\mu}(x)=\sum_{\mu \lesssim^{\prime} \lambda} \alpha^{|\lambda|-|\mu|} s_{\lambda}(x) ;
$$

that is, we sum over all partitions $\lambda$ obtained from $\mu$ by adjoining a vertical strip. On the other hand,

$$
\begin{aligned}
E_{U \in O(l)} \operatorname{det}(H(U ; x)) & =\sum_{\substack{\ell(\mu) \leq l \\
f(\mu)=0}} s_{\mu}(x) \\
E_{U \in O(l)} \operatorname{det}(U) \operatorname{det}(H(U ; x)) & =\sum_{\ell(\mu)=f(\mu)=l} s_{\mu}(x) .
\end{aligned}
$$

In each case, there is at most one way to remove a vertical strip from a generic partition to obtain one with the desired special form. Thus it remains only to determine which partitions occur. In either case, we note that $\mu_{l+1}=0$ so $\lambda_{l+1} \leq 1$; it follows that $\lambda_{2}^{\prime} \leq l$. In the second case, $\mu_{l} \geq 1$, and thus $\lambda_{1}^{\prime} \geq l$. As these conditions are readily seen to be sufficient, the result follows.

Remark. This gives another proof of Corollary 5.7, by subtracting (5.83) from 5.82) with $\alpha=1$.

It remains to consider the formulae corresponding to hyperoctahedral involutions. For an arbitrary partition $\lambda$, we define new partitions

$$
\begin{aligned}
& \lambda^{+}=\left(\lambda_{1}, \lambda_{3}, \ldots\right) \\
& \lambda^{-}=\left(\lambda_{2}, \lambda_{4}, \ldots\right) .
\end{aligned}
$$

Note that $\lambda^{-}$and $\lambda^{+}$are the unique partitions such that $\left(\lambda^{-}\right)^{2} \lesssim \lambda \lesssim\left(\lambda^{+}\right)^{2}$; also note that $f\left(\lambda^{\prime}\right)=\left|\lambda^{+}\right|-\left|\lambda^{-}\right|$.

Lemma 5.9. A partition $\lambda$ has trivial 2-core if and only if $f\left(\lambda^{+}\right)=f\left(\lambda^{-}\right)=f(\lambda) / 2$.

Proof. A partition has trivial 2-core if and only if its diagram can be tiled by dominos. By a classical result, this can happen if and only if the diagram contains as many points $(i, j)$ with $i+j$ even as with $i+j$ odd. For $a, b \in\{0,1\}$, let $C_{a b}$ be the number of points in the diagram of $\lambda$ with $(i \bmod 2, j \bmod 2)=(a, b)$. Then

$$
\begin{aligned}
& f\left(\lambda^{+}\right)=C_{11}-C_{10} \\
& f\left(\lambda^{-}\right)=C_{01}-C_{00} .
\end{aligned}
$$

But then

$$
f\left(\lambda^{+}\right)-f\left(\lambda^{-}\right)=C_{11}-C_{10}-C_{01}+C_{00}=0 .
$$


Theorem 5.10. For all $l \geq 0$,

$$
\sum_{\ell(\lambda) \leq 2 l} \alpha^{f(\lambda) / 2} \beta^{f\left(\lambda^{\prime}\right) / 2} \tilde{s}_{\lambda}(x)=\mathrm{E}_{U \in U(l)} \operatorname{det}\left((1+\alpha U)(1-\beta U)^{-1} H(U ; x) H\left(U^{\dagger} ; x\right)\right) .
$$

Proof. It follows from the lemma that for any $\lambda$ with trivial 2-core, there exist unique partitions $\mu$ and $\nu$ such that

$$
\lambda \lesssim \nu^{2} \lesssim^{\prime} 2 \mu^{2}
$$

and which satisfy

$$
\begin{gathered}
f(\lambda)=2 f(\nu)=\left|2 \mu^{2}\right|-\left|\nu^{2}\right| \\
f\left(\lambda^{\prime}\right)=\left|\nu^{2}\right|-|\lambda|
\end{gathered}
$$

Consequently,

$$
\phi_{2}\left(\sum_{\ell(\lambda) \leq 2 l}(-\alpha)^{f(\lambda) / 2} \beta^{f\left(\lambda^{\prime}\right) / 2} s_{\lambda}\right)=\phi_{2}\left(\sum_{\ell(\mu) \leq l} E^{\perp}(\sqrt{-\alpha} ; x) H^{\perp}(\sqrt{\beta} ; x) s_{2 \mu^{2}}(x)\right) .
$$

It thus suffices to show that for any partition $\mu$,

$$
\phi_{2}\left(E^{\perp}(a ; x) H^{\perp}(b ; x) s_{2 \mu^{2}}(x)\right)=s_{\mu}(x)\left(E^{\perp}\left(-a^{2} ; x\right) H^{\perp}\left(b^{2} ; x\right) s_{\mu}(x)\right),
$$

since then we may set $y=x$ in

$$
\sum_{\ell(\mu) \leq l} s_{\mu}(y)\left(E^{\perp}(\alpha ; x) H^{\perp}(\beta ; x) s_{\mu}(x)\right)=\mathrm{E}_{U \in U(l)} \operatorname{det}\left((1+\alpha U)(1-\beta U)^{-1} H(U ; x) H\left(U^{\dagger} ; y\right)\right) .
$$

By the Jacobi-Trudi identity, $s_{2 \mu^{2}}(x)$ is the determinant of the block matrix

$$
\operatorname{det}\left(B_{\mu_{i}+j-i}\right)_{1 \leq i, j \leq \ell(\mu)},
$$

where

$$
B_{m}=\left(\begin{array}{cc}
h_{2 m} & h_{2 m-1} \\
h_{2 m+1} & h_{2 m}
\end{array}\right)
$$

Equivalently, via row and column operations, we could take

$$
B_{m}=\left(\begin{array}{cc}
h_{2 m}-b h_{2 m-1} & h_{2 m-1} \\
h_{2 m+1}-(a+b) h_{2 m}+a b h_{2 m-1} & h_{2 m}-a h_{2 m-1}
\end{array}\right) .
$$

Upon applying $E^{\perp}(a ; x) H^{\perp}(b ; x)$ then $\phi_{2}$, we obtain

$$
B_{m}^{\prime}=\left(\begin{array}{cc}
h_{m} & (a+b) H^{\perp}\left(b^{2} ; x\right) h_{m-1} \\
0 & E^{\perp}\left(-a^{2} ; x\right) H^{\perp}\left(b^{2} ; x\right) h_{m}
\end{array}\right)
$$

But then

$$
\operatorname{det}\left(B_{\mu_{i}+j-i}^{\prime}\right)_{1 \leq i, j \leq \ell(\mu)}=s_{\mu}(x)\left(E^{\perp}\left(-a^{2} ; x\right) H^{\perp}\left(b^{2} ; x\right) s_{\mu}(x)\right)
$$

as required. 


\section{Corollary 5.11.}

$$
\sum_{\ell(\lambda) \leq 2 l+1} \alpha^{f(\lambda) / 2} \beta^{f\left(\lambda^{\prime}\right) / 2} \tilde{s}_{\lambda}(x)=H(\beta ; x) \mathrm{E}_{U \in U(l)} \operatorname{det}\left((1+\alpha U) H(U ; x) H\left(U^{\dagger} ; x\right)\right)
$$

Proof. Here we use the following analogue of Pieri's formula:

$$
\begin{aligned}
H(\beta ; x) \tilde{s}_{\lambda}(x) & =(-1)^{f(\lambda) / 2} \phi_{2}\left(H(\sqrt{\beta} ; x) s_{\lambda}(x)\right) \\
& =\sum_{\mu \gtrsim \lambda}(-1)^{(f(\lambda)-f(\mu)) / 2} \beta^{(|\lambda|-|\mu|) / 2} \tilde{s}_{\mu}(x) .
\end{aligned}
$$

Thus

$$
\begin{aligned}
H(\beta ; x) \sum_{\ell(\lambda) \leq l} \alpha^{f(\lambda)} \tilde{s}_{\lambda^{2}}(x) & =\sum_{\substack{\ell(\lambda) \leq l \\
\mu \gtrsim \lambda^{2}}} \beta^{|\mu| / 2-|\lambda|} \alpha^{f(\lambda)} \tilde{s}_{\mu}(x) \\
& =\sum_{\ell(\mu) \leq 2 l+1} \beta^{|\mu| / 2-\left|\mu^{-}\right|} \alpha^{f\left(\mu^{-}\right)} \tilde{s}_{\mu}(x) . \\
& =\sum_{\ell(\mu) \leq 2 l+1} \beta^{f\left(\mu^{\prime}\right) / 2} \alpha^{f(\mu) / 2} \tilde{s}_{\mu}(x) .
\end{aligned}
$$

Analogously,

Corollary 5.12. For all $l>0$,

$$
\begin{aligned}
& \sum_{\mu_{2}^{\prime} \leq 2 l} \alpha^{f(\mu) / 2} \beta^{f\left(\mu^{\prime}\right) / 2} \tilde{s}_{\mu}(x)=E(\alpha ; x) E_{U \in U(l)} \operatorname{det}\left((1-\beta U)^{-1} H(U ; x) H\left(U^{\dagger} ; x\right)\right) \\
& \left.\sum_{\mu_{2}^{\prime} \leq 2 l+1} \alpha^{f(\mu) / 2} \beta^{f\left(\mu^{\prime}\right) / 2} \tilde{s}_{\mu}(x)=E(\alpha ; x) H(\beta ; x) E_{U \in U(l)} H(U ; x) H\left(U^{\dagger} ; x\right)\right)
\end{aligned}
$$

Proof. Dualizing the proof of Corollary 5.11, we find

$$
E(\alpha ; x) \sum_{\ell(\lambda) \leq l} \beta^{f\left(\lambda^{\prime}\right)} \tilde{s}_{2 \lambda}(x)=\sum_{\mu} \alpha^{f(\mu) / 2} \beta^{f\left(\mu^{\prime}\right) / 2} \tilde{s}_{\mu}(x)
$$

where the sum is over $\mu$ such that $\left(\left(\mu^{\prime}\right)^{-}\right)^{\prime}=\lambda$ with $\ell(\lambda) \leq l$. But, as in the proof of Theorem 5.8, this condition is simply that $\mu_{2}^{\prime} \leq l$. The result follows.

\section{Pfaffian identities}

As we remarked earlier, many of the earlier proofs of the known identities from Section 5 proceeded via an intermediate pfaffian form. We sketch analogous proofs of the remaining identities of that section.

We recall the definition of the pfaffian. If $A$ is a $2 n \times 2 n$ antisymmetric matrix, the pfaffian $\operatorname{pf}(A)$ is defined by:

$$
\operatorname{pf}(A)=\frac{1}{2^{n} n !} \sum_{\pi \in S_{2 n}} \sigma(\pi) \prod_{1 \leq j \leq n} A_{\pi(2 j-1) \pi(2 j)}
$$


In the odd-dimensional case, it will be convenient to use the notation

$$
\operatorname{pf}(v ; A)
$$

to denote the pfaffian of $A$ bordered by $v$.

The fundamental identity we use is the following:

Theorem 6.1. (de Bruijn, [7) Let $X$ be a measure space, let $\rho(x, y)$ be an antisymmetric function on $X \times X$, and let $\phi_{j}(x)$ be a sequence of functions on $X$, such that for all $j$ and $k$, the function $\phi_{j}(x) \rho(x, y) \phi_{k}(y)$ is absolutely integrable. Then for $n$ even,

$$
\int_{\vec{x}} \operatorname{pf}\left(\rho\left(x_{j}, x_{k}\right)\right)_{1 \leq j, k \leq n} \operatorname{det}\left(\phi_{j}\left(x_{k}\right)\right)_{1 \leq j, k \leq n}=n ! \operatorname{pf}\left(\int_{x, y} \phi_{j}(x) \rho(x, y) \phi_{k}(y)\right)_{1 \leq j, k \leq n} .
$$

For $n$ odd,

$$
\int_{\vec{x}} \operatorname{pf}\left(\rho\left(x_{j}, x_{k}\right)\right)_{1 \leq j, k \leq n} \operatorname{det}\left(\phi_{j}\left(x_{k}\right)\right)_{1 \leq j, k \leq n}=n ! \operatorname{pf}\left(\left(\int_{x} \phi_{j}(x)\right)_{1 \leq j \leq n} ;\left(\int_{x, y} \phi_{j}(x) \rho(x, y) \phi_{k}(y)\right)_{1 \leq j, k \leq n}\right),
$$

where the integrals are all over $X$.

Proof. For $n$ even, we have:

$$
\begin{aligned}
\int_{\vec{x}} \operatorname{pf}\left(\rho\left(x_{j}, x_{k}\right)\right)_{1 \leq j, k \leq n} \operatorname{det}\left(\phi_{j}\left(x_{k}\right)\right)_{1 \leq j, k \leq n} & =\sum_{\pi_{1}, \pi_{2} \in S_{n}} \sigma\left(\pi_{1} \pi_{2}\right) \int_{\vec{x}} \prod_{1 \leq j \leq n / 2} \rho\left(x_{\pi_{1}(2 j-1)}, x_{\pi_{2}(2 j)}\right) \prod_{1 \leq j \leq n} \phi_{j}\left(x_{\pi_{2}(j)}\right) \\
& =n ! \sum_{\pi \in S_{n}} \sigma(\pi) \int_{\vec{x}} \prod_{1 \leq j \leq n / 2} \rho\left(x_{\pi(2 j-1)}, x_{\pi(2 j)}\right) \prod_{1 \leq j \leq n} \phi_{\pi(j)}\left(x_{\pi(j)}\right) \\
& =n ! \sum_{\pi \in S_{n}} \sigma(\pi) \prod_{1 \leq j \leq n / 2} \int_{x, y} \phi_{\pi(2 j-1)}(x) \rho(x, y) \phi_{\pi(2 j)}(y)
\end{aligned}
$$

For $n$ odd, we simply adjoin an extra element $\infty$ to $X$ and add a new function $\phi_{0}$ which is 0 away from $\infty$, then apply the identity for $n$ even.

Remark. As noted in [9], since the proof holds for arbitrary measure spaces, this includes the case of a sum, thus obtaining a pfaffian analogue of the Cauchy-Binet formula (which is also readily obtained as a special case). As such, this was independently rediscovered in [21], as well as some extensions (e.g., to a $q$-analogue) which we will not need. It is also worth noting that de Bruijn's formula has applications to random matrices (411).

Associated to any partition $\lambda$ of length $\leq l$, we associate a $l$-tuple $\mu_{l}(\lambda)$ of distinct integers, defined by

$$
\mu_{l}(\lambda)_{j}=\lambda_{l-j}+j
$$

with $0 \leq j<l$. In terms of this, the Jacobi-Trudi identity becomes

$$
s_{\lambda}(x)=\operatorname{det}\left(h_{\mu_{l}(\lambda)_{k}-j}(x)\right)_{0 \leq j, k<l} .
$$


We observe that this has the appropriate form for the application of Theorem 6.1, where we take

$$
\phi_{j}\left(\mu_{k}\right)=\phi_{j}\left(\mu_{k} ; x\right):=h_{\mu_{k}-j}(x) .
$$

Thus, for instance, we have

$$
\begin{aligned}
\sum_{\ell(\lambda) \leq l} s_{\lambda}(x) s_{\lambda}(y) & =\frac{1}{l !} \sum_{\mu_{1}, \mu_{2}, \ldots \mu_{l} \in \mathbb{N}} \operatorname{det}\left(\phi_{j}\left(\mu_{k} ; x\right)\right) \operatorname{det}\left(\phi_{j}\left(\mu_{k} ; y\right)\right) \\
& =\operatorname{det}\left(\sum_{\mu \in \mathbb{N}} \phi_{j}(\mu ; x) \phi_{k}(\mu ; x)\right)_{0 \leq j, k<l} \\
& =\operatorname{det}\left(g_{j-k}(x ; y)\right)_{0 \leq j, k<l} .
\end{aligned}
$$

This is, of course, precisely Gessel's original proof ([13]), slightly restated. Similarly, the identity for $\square$ follows analogously.

To handle the involution cases, we note the following: Define a function $F(\mu)$ on $l$-tuples of nonnegative integers as follows: if $\mu$ is increasing (and thus $\mu=\mu_{l}(\lambda)$ for some $\lambda$ ), then

$$
F(\mu)=\alpha^{f(\lambda)} \beta^{f\left(\lambda^{\prime}\right)} .
$$

Otherwise, $F(\mu)$ is alternating under permutations of $\mu$.

Lemma 6.2. 20] If $l$ is even, then

$$
F(\mu)=\operatorname{pf}\left(F\left(\mu_{i} \mu_{k}\right)\right)_{0 \leq i, k<l}
$$

while if $l$ is odd, then

$$
F(\mu)=-\operatorname{pf}\left(F\left(\mu_{i}\right)_{0 \leq i<l} ; F\left(\mu_{i} \mu_{k}\right)_{0 \leq i, k<l}\right)
$$

At this point, we can write

$$
\sum_{\ell(\lambda) \leq l} \alpha^{f(\lambda)} \beta^{f\left(\lambda^{\prime}\right)} s_{\lambda}(x)=\frac{1}{l !} \sum_{\mu_{1}, \mu_{2}, \ldots \mu_{l} \in \mathbb{N}} F(\mu) \operatorname{det}\left(\phi_{j}\left(\mu_{k} ; x\right)\right)_{0 \leq j, k<l} .
$$

For $l$ even, this becomes:

$$
\frac{1}{l !} \sum_{\mu_{1}, \mu_{2}, \ldots, \mu_{l} \in \mathbb{N}} \operatorname{pf}\left(F\left(\mu_{i} \mu_{k}\right)\right)_{0 \leq i, k<l} \operatorname{det}\left(\phi_{j}\left(\mu_{k} ; x\right)\right)_{0 \leq j, k<l}=\operatorname{pf}\left(\sum_{\mu, \nu} F(\mu \nu) \phi_{j}(\mu) \phi_{k}(\nu)\right)_{0 \leq j, k<l} .
$$

For $l$ odd, we must border the pfaffian with

$$
\left(-\sum_{\mu} F(\mu) \phi_{j}(\mu)\right)_{0 \leq j<l}
$$

In three cases (corresponding to $\square, \square, \bigotimes$ ), this pfaffian simplifies. We consider only $\square$ in detail (in particular since the resulting pfaffians for the other cases are more complicated).

For $l$ even, after simplifying, we find

$$
M(j, k):=\sum_{\mu, \nu} F(\mu, \nu) \phi_{j}(\mu) \phi_{k}(\nu)=M_{0}(j, k)+M_{1}(j, k),
$$


where

$$
M_{0}(j, k)=\frac{1}{2} \sum_{0 \leq d<(k-j)}\left(\left(1+\alpha^{2}\right) i_{2 d+1-(k-j)}(x)+\alpha\left(i_{2 d-(k-j)}(x)+i_{2 d+2-(k-j)}(x)\right)\right),
$$

while $M_{1}(j, k)=0$ unless $k-j$ is odd, when

$$
M_{1}(j, k)=(-1)^{j} \frac{1-\alpha^{2}}{2} H(1 ; x) H(-1 ; x)=(-1)^{j} H(1 ; \alpha, x) H(-1 ; \alpha, x)
$$

Now, $M_{1}$ has rank 2, so pf $(M)$ can be simplified via appropriate row and columns. To be precise, if we subtract row/column $i$ from row/column $i+2$ in decreasing order, $M_{1}$ is now 0 except when $(j, k)=(0,1)$ or $(1,0)$. Thus

$$
\sum_{\ell(\lambda) \leq l} \alpha^{f(\lambda)} s_{\lambda}(x)=\operatorname{pf}\left(M_{0}\right)+\frac{1}{2} H(1 ; \alpha, x) H(-1 ; \alpha, x) \operatorname{pf}\left(M_{0}^{\prime}\right),
$$

where

$$
M_{0}^{\prime}(j, k)=i_{k-j-1}(x)-i_{k-j+1}(x) .
$$

Our earlier formula follows from the following identity due to Gordon [14]:

Theorem 6.3. Let $x_{j}$ be an odd function of $j \in \mathbb{Z}$. Then

$$
\begin{gathered}
\operatorname{pf}\left(x_{k-j}\right)_{0 \leq j, k<2 l}=\operatorname{det}\left(\sum_{0 \leq m \leq k} x_{j-k+2 m+1}\right)_{0 \leq j, k<l} \\
\operatorname{pf}\left(\left(z^{j-l}\right)_{0 \leq j<2 l+1} ;\left(x_{k-j}\right)_{0 \leq k, j<2 l+1}\right)=\operatorname{det}\left(\sum_{0 \leq m \leq k}\left(z+\frac{1}{z}\right) x_{j-k+2 m+1}-\left(x_{j-k+2 m}+x_{j-k+2 m+2}\right)\right)_{0 \leq j, k<l}
\end{gathered}
$$

Remark. In addition to the proof in [14], one can also prove this by showing that the second pfaffian gives an appropriate orthogonal polynomial, or by applying a special case of de Bruijn's formula (with $X$ the unit circle).

The computation for $l$ odd is analogous.

\section{More increasing subsequence problems}

It turns out that the Schur function sums considered above can in many cases be given direct interpretations in terms of suitably defined increasing subsequences of random multisets. Let $\Omega_{1}$ and $\Omega_{2}$ be totally ordered sets, and let $W_{1} \subset \Omega_{1}$ and $W_{2} \subset \Omega_{2}$ be arbitrarily chosen subsets, with complements $\overline{W_{1}}$ and $\overline{W_{2}}$. It will also be convenient to use the corresponding indicator functions $\chi_{1}$ and $\chi_{2}$.

Definition 2. A $\left(W_{1}, W_{2}\right)$-increasing sequence in $\Omega_{1} \times \Omega_{2}$ is a sequence $\left(x_{i}, y_{i}\right)$ such that

$$
x_{i} \leq x_{i+1} \text {, and } y_{i} \leq y_{i+1},
$$

for all $i$, and such that

$$
\begin{aligned}
x_{i}=x_{i+1} & \Longrightarrow x_{i} \in W_{1}, \\
y_{i}=y_{i+1} & \Longrightarrow y_{i} \in W_{2} .
\end{aligned}
$$


Thus $W_{i}$ specifies where the $i$ th coordinate is allowed to weakly increase. In particular, a $\left(\Omega_{1}, \Omega_{2}\right)$-increasing sequence is weakly increasing, while a $(\emptyset, \emptyset)$-increasing sequence is strictly increasing. We also have a notion of a $\left(W_{1}, W_{2}\right)$-decreasing sequence, in which the inequality for $y$ is reversed.

Definition 3. A finite multiset $M$ from $\Omega_{1} \times \Omega_{2}$ is $\left(W_{1}, W_{2}\right)$-compatible if for all $i$ and $j$ such that $\chi_{1}(i) \neq \chi_{2}(j)$, $(i, j)$ occurs at most once in $M$.

If $M$ is a $\left(W_{1}, W_{2}\right)$-compatible multiset from $\Omega_{1} \times \Omega_{2}$, define $l_{W_{1} W_{2}}(M)$ to be the length of the longest $\left(W_{1}, W_{2}\right.$ )-increasing subsequence of $M$ (with, of course, the condition that an element may appear in the sequence at most as many times as it appears in $M)$. We will also require the notation $l_{W_{1} W_{2}}^{-}(M)$ for the length of the longest $\left(W_{1}, W_{2}\right)$-decreasing subsequence of $M$.

We now define, for each symmetry type, and for certain choices of $\left(W_{1}, W_{2}\right)$ for each symmetry type, a random $\left(W_{1}, W_{2}\right)$-compatible multiset, and thus a random longest increasing subsequence length. We define the multiset by specifying for each $(x, y)$ the distribution of the multiplicity $w(x, y)$ of $(x, y)$ in $M$; the multiplicities are independent unless otherwise specified. Each multiset distribution will also depend on certain parameters. For convenience, we use the following notations for the distributions which appear: $g(q)$ for the geometric distribution with parameter $q<1, b(q)$ for a variable which is 0 with probability $1 /(1+q)$ and 1 with probability $q /(1+q)$, and $g^{\prime}(\alpha, q)$ for a variable with

$$
\operatorname{Pr}\left(g^{\prime}(\alpha, q)=k\right)=\frac{1-q^{2}}{1+\alpha q} \alpha^{k \bmod 2} q^{k} .
$$

We denote the set of multisets from a set $S$ by $\mathbb{M}(S)$.

$\square$ : The parameters are subsets $W, W^{\prime} \subset \mathbb{Z}^{+}$and nonnegative sequences $q_{i}$ and $q_{i}^{\prime}$ such that: $q_{i} q_{j}^{\prime}<1$ whenever $\chi_{1}(i)=\chi_{2}(j)$ and such that

$$
Z_{W, W^{\prime}}^{\square}\left(q ; q^{\prime}\right):=\prod_{\chi(i)=\chi^{\prime}(j)}\left(1-q_{i} q_{j}^{\prime}\right) \prod_{\chi(i) \neq \chi^{\prime}(j)}\left(1+q_{i} q_{j}^{\prime}\right)^{-1} \neq 0 .
$$

$M \in \mathbb{M}\left(\mathbb{Z}^{+} \times \mathbb{Z}^{+}\right)$is chosen as follows:

$$
\begin{aligned}
& \chi(i)=\chi^{\prime}(j): w(i, j) \sim g\left(q_{i} q_{j}^{\prime}\right) \\
& \chi(i) \neq \chi^{\prime}(j): w(i, j) \sim b\left(q_{i} q_{j}^{\prime}\right)
\end{aligned}
$$

Denote the variable $l_{W W^{\prime}}(M)$ by $l_{W W^{\prime}}^{\square}\left(q ; q^{\prime}\right)$.

$\odot:$ The parameters are subsets $W, W^{\prime} \subset \mathbb{Z}$ symmetric under negation and nonnegative sequences $q_{i}$ and $q_{i}^{\prime}$ such that $q_{i} q_{j}^{\prime}<1$ whenever $\chi(i)=\chi^{\prime}(j)$ and such that

$$
Z_{W W^{\prime}}^{\square}\left(q ; q^{\prime}\right):=\prod_{\chi(i)=\chi^{\prime}(j)}\left(1-q_{i} q_{j}^{\prime}\right)^{2} \prod_{\chi(i) \neq \chi^{\prime}(j)}\left(1+q_{i} q_{j}^{\prime}\right)^{-2} \neq 0 .
$$

$M \in \mathbb{M}(\mathbb{Z} \times \mathbb{Z})$ is chosen as follows: $w(i, j)=0$ if $i=0$ or $j=0$. Otherwise:

$$
\begin{aligned}
& \chi(i)=\chi^{\prime}(j): w(i, j)=w(-i,-j) \sim g\left(q_{|i|} q_{|j|}^{\prime}\right) \\
& \chi(i) \neq \chi^{\prime}(j): w(i, j)=w(-i,-j) \sim b\left(q_{|i|} q_{|j|}^{\prime}\right)
\end{aligned}
$$

Denote the variable $l_{W W^{\prime}}(M)$ by $l_{W W^{\prime}}^{\square}\left(q ; q^{\prime}\right)$. 
$\square$ : The parameters are a subset $W \subset \mathbb{Z}^{+}$, a real number $\alpha \geq 0$ and a nonnegative sequence $q_{i}$ such that $q_{i}<1$ for all $i, \alpha q_{i}<1$ when $\chi(i)=1$, and

$$
Z_{W}^{\square}(q ; \alpha):=\prod_{\chi(i)=1}\left(1-\alpha q_{i}\right) \prod_{\chi(i)=0}\left(1+\alpha q_{i}\right)^{-1}\left(1-q_{i}^{2}\right) \prod_{\substack{i<j \\ \chi(i)=\chi(j)}}\left(1-q_{i} q_{j}\right) \prod_{\substack{i<j \\ \chi(i) \neq \chi(j)}}\left(1+q_{i} q_{j}\right)^{-1} \neq 0
$$

$M \in \mathbb{M}\left(\mathbb{Z}^{+} \times \mathbb{Z}^{+}\right)$is chosen as follows: For $i \neq j$,

$$
\begin{aligned}
& \chi(i)=\chi(j): w(i, j)=w(j, i) \sim g\left(q_{i} q_{j}\right) \\
& \chi(i) \neq \chi(j): w(i, j)=w(j, i) \sim b\left(q_{i} q_{j}\right)
\end{aligned}
$$

For $i=j$,

$$
\begin{aligned}
& \chi(i)=1: w(i, i) \sim g\left(\alpha q_{i}\right) \\
& \chi(i)=0: w(i, i) \sim g^{\prime}\left(\alpha, q_{i}\right) .
\end{aligned}
$$

Denote the variable $l_{W W}(M)$ by $l_{W}^{\square}(q ; \alpha)$.

$\nabla$ : The parameters are a subset $W \subset \mathbb{Z}^{+}$, a real number $\beta \geq 0$ and a nonnegative sequence $q_{i}$ such that $q_{i}<1$ for all $i, \beta q_{i}<1$ when $\chi(i)=0$, and

$$
Z_{W}^{\triangleright}(q ; \beta):=\prod_{\chi(i)=0}\left(1-\beta q_{i}\right) \prod_{\chi(i)=1}\left(1+\beta q_{i}\right)^{-1}\left(1-q_{i}^{2}\right) \prod_{\substack{i<j \\ \chi(i)=\chi(j)}}\left(1-q_{i} q_{j}\right) \prod_{\substack{i<j \\ \chi(i) \neq \chi(j)}}\left(1+q_{i} q_{j}\right)^{-1} \neq 0
$$

$M \in \mathbb{M}\left(\mathbb{Z}^{+} \times \mathbb{Z}^{-}\right)$is chosen as follows: For $i \neq-j$

$$
\begin{aligned}
& \chi(i)=\chi(-j): w(i,-j)=w(j,-i) \sim g\left(q_{i} q_{-j}\right) \\
& \chi(i) \neq \chi(-j): w(i,-j)=w(j,-i) \sim b\left(q_{i} q_{-j}\right)
\end{aligned}
$$

For $i=-j$,

$$
\begin{aligned}
& \chi(i)=0: w(i,-i) \sim g\left(\beta q_{i}\right) \\
& \chi(i)=1: w(i,-i) \sim g^{\prime}\left(\beta, q_{i}\right) .
\end{aligned}
$$

Denote the variable $l_{W,-W}(M)$ by $l_{W}^{\unrhd}(q ; \beta)$.

$\bigotimes$ : The parameters are a subset $W \in \mathbb{Z}$ symmetric under negation, real numbers $\alpha \geq 0$ and $\beta \geq 0$, and a nonnegative sequence $q_{i}$ such that $q_{i}, \alpha q_{i}<1$ when $\chi(i)=1, q_{i}, \beta q_{i}<1$ when $\chi(i)=0$, and

$$
Z_{W}^{\unrhd}(q ; \alpha, \beta):=\prod_{\chi(i)=0}\left(1-\beta q_{i}\right)\left(1+\alpha q_{i}\right)^{-1} \prod_{\chi(i)=1}\left(1+\beta q_{i}\right)^{-1}\left(1-\alpha q_{i}\right) Z_{W W}^{\square}(q ; q) \neq 0 .
$$

$M \in \mathbb{M}(\mathbb{Z} \times \mathbb{Z})$ is chosen as follows: $w(i, j)=0$ if $i=0$ or $j=0$. Otherwise, for $|i| \neq|j|$,

$$
\begin{aligned}
& \chi(i)=\chi(j): w(i, j)=w(-i,-j)=w(j, i)=w(-j,-i) \sim g\left(q_{|i|} q_{|j|}\right) \\
& \chi(i) \neq \chi(j): w(i, j)=w(-i,-j)=w(j, i)=w(-j,-i) \sim b\left(q_{|i|} q_{|j|}\right)
\end{aligned}
$$


for $i=j$,

$$
\begin{aligned}
& \chi(i)=0: w(i, i)=w(-i,-i) \sim g^{\prime}\left(\alpha, q_{|i|}\right) \\
& \chi(i)=1: w(i, i)=w(-i,-i) \sim g\left(\alpha q_{|i|}\right)
\end{aligned}
$$

and for $i=-j$,

$$
\begin{aligned}
& \chi(i)=0: w(i,-i)=w(-i, i) \sim g\left(\beta q_{|i|}\right) \\
& \chi(i)=1: w(i,-i)=w(-i, i) \sim g^{\prime}\left(\beta, q_{|i|}\right) .
\end{aligned}
$$

Denote the variable $l_{W W}(M)$ by $l_{W}^{\bigotimes}(q ; \alpha, \beta)$.

Remark. The conditions on the parameters are simply (1) that the various probability distributions are defined, and (2) that $M$ be finite with probability 1 . In each case the quantity $Z^{\circledast}$ gives the probability that the multiset is empty.

To relate these variables to our Schur function sums, we will need the notion of a "super-Schur" (also known as "hook Schur") function. If $x_{i}$ and $y_{i}$ are countable sets of variables, we define $s_{\lambda}(x / y)$ to be the image of $s_{\lambda}$ under the homomorphism

$$
H(t ; z) \mapsto H(t ; x / y):=H(t ; x) E(t ; y) .
$$

(See Example 3.21 of [29], but note that we have used a slightly different sign convention). In particular, since this is defined via a homomorphism, all of our identities are valid for such functions; e.g.,

$$
\sum_{\ell(\lambda) \leq l} s_{\lambda}(x / y) s_{\lambda}(z / w)=\mathrm{E}_{U \in U(l)} \operatorname{det}\left(H(U ; x) E(U ; y) H\left(U^{\dagger} ; z\right) E\left(U^{\dagger} ; w\right)\right) .
$$

Then the relation to our symmetric function identities is as follows:

Theorem 7.1. For any valid choices of parameters,

$$
\begin{aligned}
\operatorname{Pr}\left(l_{W W^{\prime}}^{\square}\left(q ; q^{\prime}\right) \leq l\right) & =Z_{W W^{\prime}}^{\square}\left(q ; q^{\prime}\right) \sum_{\ell(\lambda) \leq l} s_{\lambda}\left(q_{\bar{W}} / q_{W}\right) s_{\lambda}\left(q_{\bar{W}^{\prime}}^{\prime} / q_{W^{\prime}}^{\prime}\right) \\
\operatorname{Pr}\left(l_{W W^{\prime}}^{\square}\left(q ; q^{\prime}\right) \leq l\right) & =Z_{W W^{\prime}}^{\square}\left(q ; q^{\prime}\right) \sum_{\ell(\lambda) \leq l} \tilde{s}_{\lambda}\left(q_{\bar{W}} / q_{W}\right) \tilde{s}_{\lambda}\left(q_{\bar{W}^{\prime}}^{\prime} / q_{W^{\prime}}^{\prime}\right) \\
\operatorname{Pr}\left(l_{W}^{\square}(q ; \alpha) \leq l\right) & =Z_{W}^{\square}(q ; \alpha) \sum_{\ell(\lambda) \leq l} \alpha^{f(\lambda)} s_{\lambda}\left(q_{\bar{W}} / q_{W}\right) \\
\operatorname{Pr}\left(l_{W}^{\square}(q ; \beta) \leq l\right) & =Z_{W}^{\square}(q ; \beta) \sum_{\ell(\lambda) \leq l} \beta^{f\left(\lambda^{\prime}\right)} s_{\lambda}\left(q_{\bar{W}} / q_{W}\right) \\
\operatorname{Pr}\left(l_{W}^{\unrhd}(q ; \alpha, \beta) \leq l\right) & =Z_{W}^{\square}(q ; \alpha, \beta) \sum_{\ell(\lambda) \leq l} \alpha^{f(\lambda) / 2} \beta^{f\left(\lambda^{\prime}\right) / 2} \tilde{s}_{\lambda}\left(q_{\bar{W}} / q_{W}\right) .
\end{aligned}
$$

Remark. These processes are generalizations of processes studied by Johansson in 24]. In particular, he studies the process $l_{\mathbb{Z}^{+} \mathbb{Z}^{+}}$in the special case

$$
q_{i}=\left\{\begin{array}{ll}
\sqrt{q} & 1 \leq i \leq N \\
0 & i>N
\end{array}, \quad q_{i}^{\prime}= \begin{cases}\sqrt{q} & 1 \leq i \leq M \\
0 & i>M\end{cases}\right.
$$


as well as the process $l_{\mathbb{Z}^{+}}^{\square}$ in the special case

$$
\alpha=1, q_{i}=\left\{\begin{array}{ll}
\sqrt{q} & 1 \leq i \leq N \\
0 & i>N
\end{array} .\right.
$$

In both cases, he also studies an appropriate limit as $q \rightarrow 1$.

To prove this theorem, we will need a generalization of the Knuth correspondences [25]. Let $\Omega$ be a totally ordered set, let $W$ be a chosen subset, and let $\lambda$ be a partition.

Definition 4. An $(\Omega, W)$-bitableau $T$ of shape $\lambda$ is a function from the diagram of $\lambda$ to $\Omega$ which is weakly increasing along each row and column, such that any element of $W$ appears at most once in each column, and such that any element of $\bar{W}$ appears at most once in each row.

Remark. This is essentialy the same as the notion of bitableau given in Example 5.23 of [29]; see also [4.

We denote the set of such bitableau as $B_{\lambda}(\Omega, W)$, and observe (ibid.) that if $x_{i}$ is a sequence of indeterminates, then for any subset $W \in Z^{+}$,

$$
\sum_{T \in B_{\lambda}\left(\mathbb{Z}^{+}, W\right)} x^{T}=s_{\lambda}\left(x_{W} / x_{\bar{W}}\right)
$$

where for a bitableau $T, x^{T}$ is the product of $x_{i}^{m_{i}}$, where $i$ appears in $T m_{i}$ times.

Theorem 7.2. Given a pair $\Omega_{1}$ and $\Omega_{2}$ of totally ordered sets, and respective subsets $W_{1}$ and $W_{2}$, there exists a bijective correspondence $K_{W_{1} W_{2}}$ which, given a $\left(W_{1}, W_{2}\right)$-compatible multiset $M$, produces a pair $(P, Q)$ of bitableau of the same shape such that $P$ is a $\left(\Omega_{1}, W_{1}\right)$-bitableau and $Q$ is a $\left(\Omega_{2}, W_{2}\right)$-bitableau. A given value appears in the first (resp. second) tableau exactly as many times as it appears as the first (resp. second) coordinate in $M$.

Proof. This is essentially shown in [5]; see also [35]. While the references only deal with the case in which every element of $\bar{W}$ is greater than every element of $W$, the proofs carry over directly.

Remark 1. We have switched the order of the tableaux usually used in the Robinson-Schensted correspondence.

Remark 2. The special cases $K_{\Omega_{1} \Omega_{2}}$ and $K_{\Omega_{1} \emptyset}$ are known as the Knuth correspondence and the dual Knuth correspondence [25] respectively, while the special case $K_{\emptyset \emptyset}$ is known as the Burge correspondence [8, 12].

Since the above correspondence can be reduced to the Robinson-Schensted correspondence ([0] ), all of the usual properties carry over. We observe that the operations of inflation and deflation carry over to bitableaux, and thus one can define the dual $P^{*}$ of a bitableau. That $P^{*}$ is indeed a bitableau follows from the following:

Theorem 7.3. Let $\iota_{1}: \Omega_{1} \rightarrow \Omega_{1}^{\prime}$ and $\iota_{2}: \Omega_{2} \rightarrow \Omega_{2}^{\prime}$ be order-reversing maps; also, for a multiset $M$, define $M^{t}$ to be the multiset in $\Omega_{2} \times \Omega_{1}$ obtained by exchanging the coordinates. For any finite multiset $M \subset \Omega_{1} \times \Omega_{2}$, the 
following are equivalent:

$$
\begin{aligned}
K_{W_{1} W_{2}}(M) & =(P, Q) \\
K_{W_{1} W_{2}}\left(\left(\iota_{1} \times \iota_{2}\right)(M)\right) & =\left(\iota_{1}\left(P^{*}\right), \iota_{2}\left(Q^{*}\right)\right) \\
K_{W_{2} W_{1}}\left(M^{t}\right) & =(Q, P) \\
K_{W_{2} W_{1}}\left(\left(\iota_{2} \times \iota_{1}\right)\left(M^{t}\right)\right) & =\left(\iota_{2}\left(Q^{*}\right), \iota_{1}\left(P^{*}\right)\right) \\
K_{\overline{W_{1} W_{2}}}\left(\left(\iota_{1} \times 1\right)(M)\right) & \left.=\left(\iota_{1}\left(P^{*}\right)^{t}, Q^{t}\right)\right) \\
K_{\overline{W_{1} W_{2}}}\left(\left(1 \times \iota_{2}\right)(M)\right) & =\left(P^{t}, \iota_{2}\left(\left(Q^{*}\right)^{t}\right)\right) \\
K_{\overline{W_{2} W_{1}}}\left(\left(\iota_{2} \times 1\right)\left(M^{t}\right)\right) & =\left(\iota_{2}\left(\left(Q^{*}\right)^{t}\right), P^{t}\right) \\
K_{\overline{W_{2} W_{1}}}\left(\left(1 \times \iota_{1}\right)\left(M^{t}\right)\right) & =\left(Q^{t}, \iota_{1}\left(\left(P^{*}\right)^{t}\right)\right)
\end{aligned}
$$

Remark. For the cases $K_{\Omega_{1} \Omega_{2}}$ and $K_{\emptyset \emptyset}$, this theorem appears in [12] and [43].

When $W_{1}=W_{2}=W$ and $M=M^{t}$, the two bitableau are the same; as in the Robinson-Schensted correspondence, we can describe the number of odd-length columns:

Theorem 7.4. Let $M$ be a finite multiset in $\Omega \times \Omega$ with $M=M^{t}$. If $\lambda$ is the common shape of $K_{W W}(M)$, then $f\left(\lambda^{\prime}\right)$ is equal to the sum of (1) the number of elements of $M$ of the form $(x, x)$ with $x \in W$ and (2) the number of $x \in \bar{W}$ that appear an odd number of times in $M$.

Proof. This was known for $K_{\Omega_{1} \Omega_{2}}$ ([25]) and for $K_{\emptyset \emptyset}$ ([邽); it thus follows in general.

Let $M$ be a $\left(W_{1}, W_{2}\right)$-compatible multiset from $\Omega_{1} \times \Omega_{2}$. We observe that $l_{W_{1} W_{2}}(M) \leq l$ whenever $M$ can be written as the union of $l\left(\overline{W_{1}}, \overline{W_{2}}\right)$-decreasing sequences. (Again, we use $\left(W_{1}, W_{2}\right)$-compatibility). This motivates the notation $l_{W_{1} W_{2}}^{(k)}(M)$ for the size of the largest submultiset $M^{\prime}$ of $M$ with $l_{\overline{W_{1} W_{2}}}^{-}\left(M^{\prime}\right) \leq k$, and similarly for $l_{\frac{-(k)}{W_{1} W_{2}}}(M)$.

Theorem 7.5. Let $M$ be a finite $\left(W_{1}, W_{2}\right)$-compatible multiset from $\Omega_{1} \times \Omega_{2}$. If $\lambda$ is the common shape of $K_{W_{1} W_{2}}(M)$, then

$$
\sum_{1 \leq i \leq k} \lambda_{i}=l_{W_{1} W_{2}}^{(k)}(M), \quad \sum_{1 \leq i \leq k} \lambda_{i}^{\prime}=l_{\frac{-(k)}{W_{1} W_{2}}}(M) .
$$

Remark. For the Robinson-Schensted correspondence, this was proved in [18]; the extension to the general case is in $[5$.

In particular, $\lambda_{1}$ gives the length of the longest $\left(W_{1}, W_{2}\right)$-increasing sequence.

Proof of Theorem 7.1. We generalize the argument of [24]. Consider, for instance, the case $\square$. In this case, the probability that our random multiset $M(q ; \alpha)$ is equal to a given fixed multiset $M$ is

$$
\operatorname{Pr}(M(q ; \alpha)=M)=\operatorname{Pr}(M(q ; \alpha)=\emptyset) \alpha^{f\left(\lambda^{\prime}\right)} q^{P},
$$


where $K_{W W}(M)=(P, P)$ with $P$ of shape $\lambda$. Thus

$$
\begin{aligned}
\operatorname{Pr}\left(l_{W}^{\square}(q ; \alpha) \leq l\right) & =\sum_{\substack{\lambda_{1} \leq l \\
\lambda_{W}(M)=\lambda}} \operatorname{Pr}(M(q ; \alpha)=M) \\
& =\operatorname{Pr}(M(q ; \alpha)=\emptyset) \sum_{\substack{\ell(\lambda) \leq l \\
P \in B_{\lambda^{\prime}}\left(\mathbb{\mathbb { Z }}^{+}, W\right)}} \alpha^{f(\lambda)} q^{P} \\
& =Z_{W}^{\square}(q ; \alpha) \sum_{\ell(\lambda) \leq l} \alpha^{f(\lambda)} s_{\lambda}\left(q_{\bar{W}} / q_{W}\right) .
\end{aligned}
$$

Similar arguments hold for types $\square$ and $\square$. For $\square$ and $\otimes$, we also need the fact that $\tilde{s}_{\lambda}(x / y)$ can be defined in terms of a sum over self-dual bitableaux. Clearly, it suffices to show that the usual correspondence between self-dual tableaux and pairs of tableaux extends to bitableaux.

Define a domino $(\Omega, W)$-bitableau of shape $\lambda$ to be a tiling of the diagram of $\lambda$ with labelled dominos such that the labels increase weakly in each row and column, and such that (1) for $x \in W$, no column hits more than one domino labelled $x$, and (2) for $x \notin W$, no row hits more than one domino labelled $x$. We readily verify (using the fact that deflation preserves the bitableau property) that we have a bijection between self-dual bitableaux and domino bitableaux.

To proceed from domino bitableaux to pairs of ordinary bitableaux, it suffices to show that the usual correspondence preserves the bitableau property. But this follows from the fact that the correspondence is valid for column-strict tableaux (and thus for row-strict tableaux by symmetry), as remarked in [37.

Combining these bijections, we obtain the desired correspondence, and thus the theorem for $\square$ and $\nabla$.

Theorem 7.1 motivates the following change of notation:

$$
\begin{aligned}
l_{W W^{\prime}}^{\square}\left(q ; q^{\prime}\right) & \rightarrow l^{\square}\left(q_{\bar{W}} / q_{W} ; q_{\overline{W^{\prime}}}^{\prime} / q_{W^{\prime}}^{\prime}\right) \\
l_{W W^{\prime}}^{\square}\left(q ; q^{\prime}\right) & \rightarrow l^{\square}\left(q_{\bar{W}} / q_{W} ; q_{\overline{W^{\prime}}}^{\prime} / q_{W^{\prime}}^{\prime}\right) \\
l_{W}^{\square}(q ; \alpha) & \rightarrow l^{\square}\left(q_{\bar{W}} / q_{W} ; \alpha\right) \\
l_{W}^{\square}(q ; \beta) & \rightarrow l^{\square}\left(q_{\bar{W}} / q_{W} ; \beta\right) \\
l_{W}^{\otimes}(q ; \alpha, \beta) & \rightarrow l^{\otimes}\left(q_{\bar{W}} / q_{W} ; \alpha, \beta\right)
\end{aligned}
$$

It is somewhat startling that, even though the new notation in principle discards information, the resulting distributions are in fact exactly the same. For the involution cases, the integral formulae of Section 5 imply further that:

Corollary 7.6. For any valid parameter choices, the following pairs of random variables have the same distribution:

$$
\begin{aligned}
l^{\square}(q / r ; \alpha) & \sim l^{\square}(q / \alpha, r ; 0), \\
\left\lfloor\frac{1}{2} l^{\square}(q / r ; \beta)\right\rfloor & \sim \frac{1}{2} l^{\nabla}(q / r ; 0), \\
\left\lceil\frac{1}{2} l^{\square}(q / r ; \beta)\right\rceil & \sim \frac{1}{2} l^{\nabla}(\beta, q / r ; 0),
\end{aligned}
$$




$$
\begin{aligned}
& \left\lfloor\frac{1}{2} l^{\otimes}(q / r ; \alpha, \beta)\right\rfloor \sim l^{\square}(q / \alpha, r ; q / r) \\
& \left\lceil\frac{1}{2} l^{\varpi}(q / r ; \alpha, \beta)\right\rceil \sim l^{\square}(\beta, q / \alpha, r ; q / r)
\end{aligned}
$$

where, for instance $l^{\square}(\alpha, q / r ; 0)$ corresponds to a process in which $\alpha$ has been inserted into the sequence $q$.

Proof. We have:

$$
\begin{aligned}
\operatorname{Pr}\left(l^{\square}(q / r ; \alpha) \leq l\right) & =Z^{\square}(q / r ; \alpha) \mathrm{E}_{U \in O(l)} \operatorname{det}((1+\alpha U) H(U ; q / r)) \\
& =Z^{\square}(q / \alpha, r ; 0) \mathrm{E}_{U \in O(l)} \operatorname{det}(H(U ; q / \alpha, r))
\end{aligned}
$$

and similarly for the other cases.

Remark 1. By the arguments of Theorem 5.6, one can prove something stronger. Namely, the joint distribution of the lengths of the odd-numbered rows is the same for $\square(q / r ; \alpha)$ and for $\square(q / \alpha, r ; 0)$, and similarly for the other cases. For the even-numbered rows, the argument of Theorem 5.8 shows that the joint distribution is independent of $\alpha$. In the Laguerre limit (see below), we obtain the following fact. Consider the "matrix ensemble" with joint eigenvalue density $($ on $[0, \infty))$ proportional to

$$
\operatorname{pf}\left(\operatorname{sgn}\left(x_{k}-x_{j}\right) e^{A\left|x_{k}-x_{j}\right|}\right)_{1 \leq j, k \leq 2 n} \prod_{1 \leq j<k \leq 2 n}\left(x_{k}-x_{j}\right) \prod_{1 \leq j \leq 2 n} e^{-C x_{j}}
$$

where $A$ and $C$ are parameters with $C>\max (A, 0)$; note that if $x_{1}<x_{2}<\ldots x_{2 n}$, then

$$
\operatorname{pf}\left(\operatorname{sgn}\left(x_{k}-x_{j}\right) e^{A\left|x_{k}-x_{j}\right|}\right)_{1 \leq j, k \leq 2 n}=\exp \left(A \sum_{1 \leq j \leq 2 n}(-1)^{j} x_{2 j}\right) .
$$

Then the joint distribution of the second, fourth, sixth, etc. largest eigenvalues is independent of $A$. Since for $A=0$ this ensemble is the Laguerre orthogonal ensemble (LOE), while in the limit $A \rightarrow-\infty$, it becomes the Laguerre symplectic ensemble (LSE), we find in particular that the distribution of the second-largest eigenvalue of LOE is the same as the distribution of the largest eigenvalue of LSE (since every eigenvalue of LSE occurs twice). For an alternate proof, and generalizations, see [1].

Remark 2. We can recover Theorems 1.2 and 4.2 from Theorem 7.1 by taking suitable limits. For instance, for $\square$, we take $q_{i}=q_{i}^{\prime}=t / N$ for $1 \leq i \leq N$, and $q_{i}=q_{i}^{\prime}=0$ otherwise. As $N \rightarrow \infty$, the resulting point process converges to the usual Poisson process. In Corollary 7.6, if we take the corresponding limit for the right-hand-sides, we obtain Poisson processes in which, instead of adding extra diagonal points, we add extra side points. Thus we obtain the fact that these two Poisson processes have exactly the same distribution. For instance, if $n$ and $m$ are nonnegative integers, the distribution of the longest weakly increasing subsequence is the same if we choose either: Pick $n$ points at random in the triangle $0 \leq y \leq x \leq 1$, and $m$ points at random with $0 \leq x=y \leq 1$; or: Pick $n$ points at random in the triangle $0 \leq y \leq x \leq 1$, and $m$ points at random with $y=0$ and $0 \leq x \leq 1$. It is not at all clear why these distributions should be the same. 
Remark 3. By taking a Poisson limit for only some of the variables, we obtain increasing subsequence interpretations for the image of the Schur function sums under homomorphisms of the form

$$
H(t ; z) \mapsto e^{a t} H(t ; x) E(t ; y)
$$

with $a, x_{i}$ and $y_{i}$ nonnegative (and satisfying the appropriate additional conditions). As this is the most general case in which the images of $s_{\lambda}$ are all nonnegative ([40]), this is presumably the most general case for which such an interpretation can be given.

Remark 4. In addition to the Poisson limit, another natural limit is the Laguerre limit (24]), in which the random multiplicities are exponentially distributed. More precisely, one fixes integers $N$ (and $N^{\prime}$ as necessary), and considers a process in which $q$ and $q^{\prime}$ are constant on the respective intervals $[1, N]$ and $\left[1, N^{\prime}\right]$, and 0 otherwise, then takes the limit $q, q^{\prime} \rightarrow 1$. In the case $\alpha=\beta=0$, one finds the following curious fact, analogous to Theorem 1.2 .

Given a complex $N \times N^{\prime}$ matrix $M$, we define two decreasing sequences of nonnegative real numbers. $\Sigma(M)_{i}$ is the $i$ th largest eigenvalue of $M M^{\dagger}$, or 0 if $i>\min \left(N, N^{\prime}\right)$, while $\Delta(M)_{i}$ is defined so that

$$
\sum_{1 \leq i \leq j} \Delta(M)_{i}=\max _{S} \sum_{(k, l) \in S}\left|M_{k l}\right|^{2}
$$

where $S$ ranges over unions of $j$ decreasing paths.

Theorem 7.7. Let $N$ be a positive even integer. Map $H$ (recall Section 目) into transformations of $N \times N$ complex matrices as follows:

$$
/ \mapsto\left(M \mapsto-M^{t}\right) \quad \text { and } \quad \backslash \mapsto\left(M \mapsto J M^{t} J\right)
$$

Let $G^{\circledast}(N)$ be the Gaussian distribution on matrices with the appropriate symmetry. Then for each $\circledast$, the distributions $\Sigma\left(G^{\circledast}(N)\right)$ and $\Delta\left(G^{\circledast}(N)\right)$ are the same.

Proof. (Sketch) That we can compute the distribution of $\Delta\left(G^{\circledast}(N)\right)$ (as well as the significance of this result) follows from the fact that it is the Laguerre limit of the appropriate discrete process with symmetry $\circledast$. Thus, as in 24 for $\square$, we find that the appropriate sum of pfaffians (see Section 6 ) tends to a Riemann integral.

On the other hand, the distributions $G^{\circledast}(N)$ for $\square, \nabla$, and $\nabla$ are well-studied (these are unconstrained, antisymmetric, and symmetric complex matrices, respectively), and in particular the distribution $\Sigma\left(G^{\circledast}(N)\right)$ is known in these cases (see 11]). For $\square$ and $₫$, we can perform simple row and column operations (not changing $\Sigma(M))$ to reduce to the case of $\square$.

We find that the two density functions we obtain are the same, proving the theorem.

Remark. For $\square$ and $\square, N$ need not be even, while for $\square$ and $\square$, the matrices need not be square.

\section{Invariants and increasing subsequences}

Consider the integral

$$
\mathrm{E}_{U \in U(l)}|\operatorname{Tr}(U)|^{2 n}
$$


The function $|\operatorname{Tr}(U)|^{2 n}$ is the character of a representation of $U(l)$, since

$$
|\operatorname{Tr}(U)|^{2 n}=\operatorname{Tr}\left(U^{\otimes n} \otimes \bar{U}^{\otimes n}\right),
$$

where we write $A^{\otimes n}$ for the tensor product of $A$ with itself $n$ times. It follows that (8.1) (and thus $f_{n l}^{\square}$ ) gives the dimension of the fixed subspace of that representation. Equivalently, this is the dimension of the space $C_{n}(U(l))$ of operators on $\left(\mathbb{C}^{l}\right)^{\otimes n}$ that commute with $U^{\otimes n}$ for all $U \in U(l)$.

Given a permutation $\pi \in S_{n}$, we can associate an operator $T_{l}(\pi)$ on $\left(\mathbb{C}^{l}\right)^{\otimes n}$ as follows:

$$
T_{l}(\pi)\left(v_{1} \otimes v_{2} \otimes \ldots v_{n}\right)=\left(v_{\pi(1)} \otimes v_{\pi(2)} \otimes \ldots v_{\pi(n)}\right) .
$$

This operator clearly commutes with $U^{\otimes n}$ for $U \in U(l)$, and thus $T_{l}$ extends to a map from $\mathbb{C}\left[S_{n}\right]$ to $C_{n}(U(l))$. Indeed, this map is known to be surjective (see, e.g., [7]); i.e., the operators $T_{l}(\pi)$ span $C_{n}(U(l))$. In general, however, it is not injective. For any subset $S \subset\{1,2, \ldots n\}$, define two elements of $\mathbb{C}\left[S_{n}\right]$ :

$$
\begin{aligned}
E_{S} & :=\sum_{\substack{\pi \in S_{n} \\
\pi(x)=x, \forall x \notin S}} \sigma(\pi) \pi \\
H_{S} & :=\sum_{\substack{\pi \in S_{n} \\
\pi(x)=x, \forall x \notin S}} \pi .
\end{aligned}
$$

Lemma 8.1. For $l \geq n, T_{l}$ is injective on $\mathbb{C}\left[S_{n}\right]$, while for $l<n$, the kernel of $T_{l}$ contains all elements of the form $\pi E_{S}$ with $|S|>l$.

Proof. That $T_{l}$ is injective for $l \geq n$ is straightforward; we simply observe that if $v_{1}, v_{2}, \ldots v_{n}$ are linearly independent vectors, then the vectors

$$
T_{l}(\pi)\left(v_{1} \otimes v_{2} \otimes \ldots v_{n}\right)=v_{\pi(1)} \otimes v_{\pi(2)} \otimes \ldots v_{\pi(n)}
$$

are linearly independent, as $\pi$ ranges over $S_{n}$.

Thus, suppose $l<n$. That $\pi E_{S} \in \operatorname{ker} T_{l}$ follows from the fact that any tensor product of $|S|>l$ basis vectors must contain at least one basis vector more than once, so will be taken to 0 by $E_{S}$.

Remark. It follows from the proof of Theorem 8.2 below that these elements also span the kernel.

Using this fact, we obtain:

Theorem 8.2. For any nonnegative integers $l$ and $n$, the set

$$
\left\{T_{l}(\pi): \pi \in S_{n} \mid \ell^{-}(\pi) \leq l\right\}
$$

is a basis of $C_{n}(U(l))$.

Proof. We first need to show that, given any permutation $\pi$ with a long decreasing subsequence, we can express $T_{l}(\pi)$ as a linear combination of $T_{l}\left(\pi^{\prime}\right)$ with $\pi^{\prime}$ ranging over permutations without long decreasing subsequences. 
Let $\pi$ be such a permutation, and let $S$ be a subset of size $l+1$ on which $\pi$ is decreasing. By the lemma, it follows that

$$
\begin{aligned}
T_{l}(\pi) & =T_{l}\left(\pi-\pi E_{S}\right) \\
& =-\sigma(\pi) \sum_{\substack{\pi^{\prime} \in S_{n} \\
\pi^{\prime}(x)=\pi(x), \forall x \notin S \\
\pi^{\prime} \neq \pi}} \sigma\left(\pi^{\prime}\right) T_{l}\left(\pi^{\prime}\right) .
\end{aligned}
$$

Now each permutation $\pi^{\prime}$ on the right hand side agrees with $\pi$ outside $S$, but is no longer decreasing on $S$. It follows that each $\pi^{\prime}$ has strictly fewer inversions than $\pi$ (reduce to the case in which $\pi^{\prime}$ differs from $\pi$ by a 2-cycle).

It follows that if we iterate this reduction, we will eventually obtain a linear combination of permutations that cannot be reduced. But this is precisely the desired result.

It remains only to show that the elements $T_{l}(\pi)$ are linearly independent, which we will do via a triangularity argument. If we choose a basis of $V$, we can view $T_{l}$ as the restriction to $\{1,2, \ldots l\}^{n}$ of the corresponding action of $S_{n}$ on the set $W_{n}=\mathbb{N}^{n}$. Now, to a given permutation $\pi$, we associate two words $w_{1}(\pi)$ and $w_{2}(\pi)$ as follows: $w_{1}(\pi)_{j}$ is equal to the length of the longest decreasing subsequence of $\pi$ starting with $j$; similarly $w_{2}(\pi)_{j}$ is equal to the length of the longest decreasing subsequence of $\pi$ starting in position $j$. (Since $\pi$ has longest decreasing subsequence of length $\leq l$, these are indeed in $\{1,2, \ldots l\}^{n}$.) We easily see that $w_{2}(\pi)=\pi\left(w_{1}(\pi)\right)$, and thus that $T_{l}(\pi)$ has coefficient 1 on the pair $\left(w_{1}(\pi), w_{2}(\pi)\right)$. The claim is then that any other permutation taking $w_{1}(\pi)$ to $w_{2}(\pi)$ has strictly more inversions than $\pi$.

Define the number of inversions $i(w)$ of a word $w$ to be the number of coordinate positions $i<j$ such that $w_{j}<w_{i}$. By standard arguments, we find that if $\pi(v)=w$, then $i(w) \leq i(v)+i(\pi)$; equality holds if for any pair $i<j$ such that $\pi_{i}>\pi_{j}$, we have $v_{i}<v_{j}$ and $w_{i}>w_{j}$. We immediately deduce that (a) $i\left(w_{2}(\pi)\right)=i\left(w_{1}(\pi)\right)+i(\pi)$ and (b) if $\pi^{\prime}\left(w_{1}(\pi)\right)=w_{2}(\pi)$, then $i\left(\pi^{\prime}\right) \geq i(\pi)$. Finally, if $\pi^{\prime}\left(w_{1}(\pi)\right)=w_{2}(\pi)$ with $i\left(\pi^{\prime}\right)=i(\pi)$, then $\pi^{\prime}$ has not only the same number of inversions as $\pi$, but indeed the same $s$ et of inversions; it follows that $\pi^{\prime}=\pi$.

Probably the most important consequence of Theorem 8.2 is that it gives an elementary proof that

Corollary 8.3. For any integers $n$ and $l$,

$$
E_{U \in U(l)}|\operatorname{Tr}(U)|^{2 n}=\operatorname{dim}\left(C_{n}(U(l))\right)=f_{n l} .
$$

Remark 1. A reduction algorithm closely related to that used above appeared in [34], for an application to P.I. algebras. The connection to invariant theory, as well as the various generalizations given below appear to be new, however.

Remark 2. A different basis for $l=2$ (based on one of the many combinatorial interpretations of the Catalan numbers) was given in [17].

Remark 3. We could, of course, just as easily have used the permutations without long increasing subsequences to form the basis. The current choice has the merit of giving a basis containing the identity and closed under taking inverses, as well as making the proof of linear independence somewhat cleaner. 
Remark 4. While we defined everything over $\mathbb{C}$, we observe that both the representation $T_{l}$ and the above reduction algorithm are actually defined over $\mathbb{Z}$.

Remark 5. There are also, of course, analogues of Corollary 8.3 associated with Theorems 8.4 through 8.8; we leave the details to the reader.

More generally, Theorem 7.1 implies

$$
Z_{W W^{\prime}}^{\square}\left(q ; q^{\prime}\right)^{-1} \operatorname{Pr}\left(l_{W W^{\prime}}^{\square}\left(q ; q^{\prime}\right) \leq l\right)=E_{U \in U(l)} \operatorname{det}\left(H\left(U ; q_{\bar{W}} / q_{W}\right) H\left(U^{\dagger} ; q_{\overline{W^{\prime}}}^{\prime} / q_{W^{\prime}}^{\prime}\right)\right) .
$$

Consider the coefficient of a monomial

$$
\prod_{i} q_{i}^{\nu_{i}}\left(q_{i}^{\prime}\right)^{\nu_{i}^{\prime}}
$$

in the right-hand-side. By the properties of $H(t ; x / y)$, this is

$$
E_{U \in U(l)} \prod_{i \in W} e_{\nu_{i}}(U) \prod_{i \in \bar{W}} h_{\nu_{i}}(U) \prod_{i \in W^{\prime}} \overline{e_{\nu_{i}^{\prime}}(U)} \prod_{i \in \overline{W^{\prime}}} \overline{h_{\nu_{i}^{\prime}}(U)}
$$

Again, this is the expectation of a character, and thus computes the dimension of a space of invariants. The corresponding coefficient of the left-hand-side counts $\left(W, W^{\prime}\right)$-compatible multisets without $\left(W, W^{\prime}\right)$-increasing subsequences of length $l+1$, in which $i$ appears $\nu_{i}$ times as a first coordinate and $\nu_{i}^{\prime}$ times as a second coordinate. Thus to obtain the analogue of Theorem 3.2, we first need an analogue of $T_{l}$ for multisets.

Given a composition $\nu$, we define a partition $S(\nu)$ of $\{1,2, \ldots|\nu|\}$ by

$$
S(\nu)_{i}=\left\{j: \sum_{k<i} \nu_{k}<j \leq \sum_{k \leq i} \nu_{k}\right\}
$$

Associated to this partition is an operator

$$
\Pi(\nu)=\prod_{i \in W} E_{S(\nu)_{i}} \prod_{i \in \bar{W}} H_{S(\nu)_{i}}
$$

Then the representation of $U(l)$ in question is simply the action by conjugation of $U^{\otimes n}$ on operators of the form $T_{l}\left(\Pi\left(\nu^{\prime}\right)\right) A T_{l}(\Pi(\nu))$. In particular, the invariant subspace is spanned by operators of the form $T_{l}\left(\Pi\left(\nu^{\prime}\right) \pi \Pi(\nu)\right)$ with $\pi \in S_{n}$. We note that if two elements of $S_{i}$ with $i \in W$ each map to elements of the same $S_{j}^{\prime}$ with $j \notin W^{\prime}$, then $\Pi\left(\nu^{\prime}\right) \pi \Pi(\nu)=0$; and similarly if $i \notin W$ and $j \in W^{\prime}$. It follows that operators of the form $\Pi\left(\nu^{\prime}\right) \pi \Pi(\nu)$ are, up to sign, in one-to-one correspondence with $\left(W, W^{\prime}\right)$-compatible multisets with content $\left(\nu, \nu^{\prime}\right)$. In particular, given a $\left(W, W^{\prime}\right)$-compatible multiset $M$ with content $\left(\nu, \nu^{\prime}\right)$, we obtain a element of $\mathbb{C}\left[S_{n}\right]$ which we denote $T_{l}(M)$. Writing $\mathcal{M}_{W W^{\prime}}^{\square}\left(\nu ; \nu^{\prime}\right)$ for the set of such multisets,

Theorem 8.4. For any nonnegative integers $l$ and compositions $\nu$ and $\nu^{\prime}$,

$$
\left\{T_{l}(M): M \in \mathcal{M}_{W W^{\prime}}^{\square}\left(\nu ; \nu^{\prime}\right) \mid \ell_{W W^{\prime}}^{-}(M) \leq l\right\}
$$

is a basis of $\Pi\left(\nu^{\prime}\right) C_{n}(U(l)) \Pi(\nu)$. 
Proof. Given a multiset $M$ in $\mathbb{Z}^{+} \times \mathbb{Z}^{+}$, we define an inversion of $M$ to be a pair of elements $(x, y) \in M$, $(z, w) \in M$ with $x<z$ and $y>w$ (i.e., a strictly decreasing subsequence of length 2).

Now, suppose $M \in \mathcal{M}_{W W^{\prime}}\left(n ; n^{\prime}\right)$ has a $\left(W, W^{\prime}\right)$-decreasing subsequence of length $l+1$. Choose a permutation $\pi$ corresponding to $M$, and let $S \subset\{1,2, \ldots n\}$ be the set of positions of $\pi$ corresponding to the $\left(W, W^{\prime}\right)$-decreasing subsequence. As before, we have

$$
T\left(\Pi\left(\nu^{\prime}\right) \pi E_{S} \Pi(\nu)\right)=0 .
$$

Now, we readily see that for any permutation that appears in the left-hand-side, the corresponding multiset has at most as many inversions as $M$. Indeed, the number of inversions is strictly smaller unless the corresponding multiset is equal to $M$. Thus it remains only to show that the terms corresponding to $M$ do not cancel. The only way that the term

$$
\sigma\left(\pi^{\prime}\right) \Pi\left(\nu^{\prime}\right) \pi \pi^{\prime} \Pi(\nu)
$$

can correspond to $M$ is if we can write

$$
\pi^{\prime}=\left(\prod_{i} \pi^{-1} \pi_{1 i}^{\prime} \pi\right)\left(\prod_{i} \pi_{2 i}^{\prime}\right)
$$

where $\pi_{2 i}^{\prime}$ fixes the complement of $S \cap S(\nu)_{i}$ and $\pi_{1 i}^{\prime}$ fixes the complement of $\pi(S) \cap S\left(\nu^{\prime}\right)_{i}$. Now, by the definition of $\left(W, W^{\prime}\right)$-increasing subsequence, $S \cap S(\nu)_{i}$ contains at most one element when $i \notin W$; similarly $\pi(S) \cap S\left(\nu^{\prime}\right)_{i}$ contains at most one element when $i \notin W^{\prime}$. In other words, we can write

$$
\pi^{\prime}=\left(\prod_{i \in W^{\prime}} \pi^{-1} \pi_{1 i}^{\prime} \pi\right)\left(\prod_{i \in W} \pi_{2 i}^{\prime}\right)
$$

But then

$$
\sigma\left(\pi^{\prime}\right) \Pi^{\prime} \pi \pi^{\prime} \Pi=\Pi^{\prime} \pi \Pi
$$

as desired.

The proof of linear independence is analogous to that in Theorem 8.2. Of the permutations associated to $M$, there is a unique one $(\pi(M))$ such that each element of $W$ and $W^{\prime}$ induces a decreasing subsequence and each element of $\bar{W}$ and $\overline{W^{\prime}}$ induces an increasing subsequence. We define $w_{1}(M)=w_{1}(\pi(M)), w_{2}(M)=w_{2}(\pi(M))$, and observe that any other multiset $M^{\prime}$ with a nonzero coefficient at $\left(w_{1}(M), w_{2}(M)\right)$ satisfies $i\left(\pi\left(M^{\prime}\right)\right)>$ $i(\pi(M))$.

Remark 1. It is possible to renormalize the basic invariants $T_{l}(M)$ in such a way that the reduction algorithm is integral. We need simply divide $T_{l}(M)$ by

$$
\prod_{i \in W, j \in W^{\prime}}\left|S(\nu)_{i} \cap \pi\left(S\left(\nu^{\prime}\right)_{j}\right)\right| !,
$$

where $T_{l}(M)=\Pi\left(\nu^{\prime}\right) \pi \Pi(\nu)$. 
Remark 2. The space $\Pi\left(\nu^{\prime}\right) C_{n}(U(l)) \Pi(\nu)$ can be thought of as the space of simultaneous (multilinear) invariants of a collection of symmetric and antisymmetric tensors, some covariant and some contravariant. Of special interest is the case in which $\nu^{\prime}$ is the composition $l^{k}$, with $1,2, \ldots k \in W^{\prime}$. In this case, the space

$$
\left(E_{\{1,2, \ldots l\}} E_{\{l+1, l+2, \ldots 2 l\}} \ldots\right) C_{n}(U(l)) \Pi(\nu)
$$

corresponds to relative invariants of a collection of symmetric and antisymmetric covariant tensors; i.e., transforming the tensors multiplies the invariant by a power of the determinant. There is a known algorithm (the straightening algorithm [36]) for computing a basis of such invariants. In fact, we observe that the resulting basis is, up to constant factors, the same as our basis. Thus our algorithm can be viewed as a generalization of this algorithm (different from the generalization to the "fourfold" algebra of [19]). Similarly, the algorithms below for the orthogonal and symplectic groups can be thought of as straightening algorithms for those groups.

Remark 3. There is also a "quantum" analogue of this result. If one replaces the unitary group $U(l)$ by the quantum enveloping algebra $U_{q}\left(\mathfrak{g} l_{l}\right)$, the role of the symmetric group is now played by the Hecke algebra [22]. As long as $S$ consists of consecutive elements, there is no difficulty in defining $E_{S}$ and $H_{S}$ (these are idempotents corresponding to 1-dimensional characters of parabolic subalgebras). We find that the kernel of the quantum $T_{l}$ is the ideal generated by $E_{S}$ with $S=\{k, k+2, \ldots k+l\}, 1 \leq k \leq n-l$. Using the appropriate normalization, we obtain a reduction algorithm integral over $\mathbb{Z}[q]$. (We also have linear independence whenever $q$ is not a root of unity.) Of particular interest is the "crystal limit" $q=0$. Under that specialization, the relations take the form $T_{l}(M)=0$, with $M$ ranging over multisets with long decreasing subsequences. This case surely merits further investigation, given the connection [28] between the crystal limit of the quantum straightening algorithm and the Robinson-Schensted-Knuth correspondence. It would also be interesting to understand the analogues for the quantum orthogonal and symplectic groups.

For the orthogonal group $O(l)$, there is a "basic" invariant $T_{l}^{\square}(\tau)$ associated to any fixed-point-free involution $\tau$ in $S_{2 n}$ such that the basic invariants span the invariant space of $U^{\otimes 2 n}$ (which we denote by $F_{2 n}(O(l)$ ). These transform under $T_{l}(\pi)$ as:

$$
T_{l}(\pi) T_{l}^{\square}(\tau)=T_{l}^{\square}\left(\pi^{-1} \tau \pi\right) .
$$

We write $\pi \cdot \tau$ for the corresponding action on the formal span of fixed-point-free involutions. For any composition $\nu$ and nonnegative integer $a$, we define

$$
\mathcal{M}_{W}^{\square}(\nu ; a)
$$

to be the set of symmetric ( $W, W$ )-compatible multisets with content composition $\nu$ and with $a$ "fixed points" (i.e., $(i, i) \in M$ such that $i \in W$, together with $(i, i)$ with $i \notin W$ such that $(i, i)$ has odd multiplicity.) Clearly, there is a correspondence (up to sign) between $\mathcal{M}_{W}^{\square}\left(n_{i} ; 0\right)$ and elements of the form $\Pi \cdot \tau$.

Theorem 8.5. For any nonnegative integer $l$ and any composition $\nu$,

$$
\left\{T_{l}^{\square}(M): M \in \mathcal{M}_{W}^{\nabla}(\nu ; 0) \mid \ell_{W W}(M) \leq l\right\}
$$

is a basis of $\Pi(\nu) F_{2 n}(O(l))$. 
Proof. The argument is analogous. In eliminating a given increasing subsequence, we replace both it and its reflection through the diagonal by non-increasing subsequences, so the number of inversions increases.

The proof of linear independence is analogous to that of Theorem 8.4. We simply switch the reverse the inequalities on the second coordinates before applying the above arguments.

This corresponds to taking the coefficient of a monomial $q^{\nu}$ in the identity

$$
Z_{W}^{\square}(q ; \alpha)^{-1} \operatorname{Pr}\left(l_{W}^{\square}(q ; \alpha) \leq l\right)=E_{U \in O(l)} \operatorname{det}\left(H\left(U ; q_{\bar{W}} / \alpha, q_{W}\right)\right)
$$

To handle the general case, we need to map an element of $M_{W}^{\square}(\nu ; a)$ (corresponding to the monomial $q^{\nu} \alpha^{a}$ ) to an element of

$$
\Pi^{\square}(\nu ; a) F_{2 n}(O(l))
$$

where we define

$$
\Pi^{\square}(\nu ; a)=\Pi(\nu) E_{\{|\nu|+1,|\nu|+2, \ldots|\nu|+a\}} .
$$

But this is simple: simply convert each fixed point $(i, i)$ to a pair of elements $(i, \infty)$ and $(\infty, i)$, and apply $T_{l}^{\square}$.

Theorem 8.6. For any nonnegative integers $l$ and $a$ and any composition $\nu$,

$$
\left\{T_{l}^{\square}(M): M \in \mathcal{M}_{W}^{\square}(\nu ; a) \mid \ell_{W W}(M) \leq l\right\}
$$

is a basis of $\Pi^{\square}(\nu ; a) F_{2 n}(O(l))$.

Proof. The main difficulty here is that the naïve extension of the above algorithm is no longer guaranteed to terminate; for instance, corresponding to the increasing subsequence 13 of 132 , we have the identity

$$
T_{2}^{\square}(132)=T_{2}^{\square}(213)
$$

Since 13 is an increasing subsequence of both sides, we could clearly loop indefinitely.

The solution is to require that the increasing subsequence consist entirely of points $(x, y)$ with $x \leq y$. Even with this restriction, the above proof does not entirely carry over; for instance, both 132 and 213 have the same number of inversions (i.e., 1).

For an involution $\tau$, denote by $S_{\leq}(\tau)$ the set of $i$ with $i \leq \tau(i)$. Given a multiset $M$, we then define $M_{\leq}(M)$ to be the multiset corresponding to $S_{\leq}(\tau)$ where $\tau$ corresponds to $M$. Given two multisets $M_{1}$ and $M_{2}$ of the same size on the same totally ordered set, we write $M_{1} \leq M_{2}$ to indicate that we can identify elements of $M_{1}$ and $M_{2}$ in such a way that each element of $M_{1}$ is $\leq$ the corresponding element of $M_{2}$. Then the theorem follows from the following observation:

If $M^{\prime}$ is one of the multisets obtained after eliminating an increasing subsequence of $M$, then $M_{\leq}\left(M^{\prime}\right) \leq$ $M_{\leq}(M)$. If equality occurs, then either $M^{\prime}=M$ or $M^{\prime}$ has strictly more inversions than $M$.

Linear independence follows as above. 
For the symplectic group $S p(2 l)$, the basic invariants again correspond to involutions, but now transform under $T_{l}(\pi)$ as:

$$
T_{2 l}(\pi) T_{l}^{\square}(\tau)= \pm T_{2 l}^{\unrhd}\left(\pi^{-1} \tau \pi\right),
$$

with

$$
T_{2 l}(\pi) T_{l}^{\square}(\tau)=\sigma(\pi) T_{l}^{\square}(\tau)
$$

whenever $\pi$ commutes with $\tau$. For any composition $\nu$ and nonnegative integer $b$, we define

$$
\mathcal{M}_{W}^{\triangleright}(\nu ; b)
$$

to be the set of symmetric $(\bar{W}, \bar{W})$-compatible multisets with content composition $\nu$ and with $b$ fixed points.

Theorem 8.7. For any nonnegative integer $l$ and composition $\nu$,

$$
\left\{T_{l}^{\square}(M): M \in \mathcal{M}_{W}^{\triangleright}(\nu ; 0) \mid \ell_{W W}^{-}(M) \leq 2 l\right\}
$$

is a basis of $\Pi(\nu) F_{2 n}(S p(2 l))$.

Proof. Analogous. The only issue is that the long decreasing subsequence we eliminate must be symmetric about the diagonal (clearly always possible).

For linear independence, we choose a symplectic basis of $V$ indexed $v_{ \pm 1}, v_{ \pm 2}$. We thus find that the nonzero coefficients of an involution $\tau$ correspond to words of length $2 n$ on $\pm \mathbb{Z}^{+}$such that $\tau(w)=-w$. The word $w(\tau)$ associated to an involution is now defined so that $\left|\tau_{j}\right|$ is equal to half the length of the longest symmetric decreasing subsequence starting or ending with $j$; the sign is positive if the sequence ends with $j$, and negative otherwise. Other than this, the arguments are analogous.

This extends easily to the case when fixed points are allowed; in this case, we define

$$
\Pi_{2 l}^{\triangleright}(\nu ; b)=\Pi(\nu) H_{|\nu|+1,|\nu|+2, \ldots|\nu|+b} .
$$

When eliminating (symmetric) decreasing subsequences, if the subsequence has even length, we can proceed as above; otherwise, we permute only those elements not corresponding to $\infty$. In either case, we convert an decreasing subsequence to a non-decreasing subsequence. (And the linear independence argument carries over.) Thus

Theorem 8.8. For any nonnegative integers $l$ and $b$ and any composition $\nu$,

$$
\left\{T_{l}^{\triangleright}(M): M \in \mathcal{M}_{W}^{\nabla}(\nu ; b) \mid \ell_{W W}^{-}(M) \leq 2 l\right\}
$$

is a basis of $\Pi^{\triangleright}(\nu ; b) F_{2 n}(S p(2 l))$.

It is not entirely clear how to proceed for either of $\square$ or $\otimes$. For $\square$, or more generally $U(p) \times U(q)$, the centralizer algebra corresponds as expected to a certain representation $T_{p, q}$ of the hyperoctahedral group, in 
which permutations are mapped via $T_{p+q}$, and sign changes correspond to an operator with eigenvalues $1(p$ times) and -1 ( $q$ times). Given a set $S \subset\{1,2, \ldots n\}$, we can define two elements $E_{S}^{ \pm} \in \mathbb{C}\left[H_{n}\right]$. Each is a sum over elements of $H_{n}$ that fix the complement of $S$; in $E^{+}$, we multiply by the sign of the corresponding permutation, while in $E^{-}$, we further multiply by the number of sign changes. Since the group $U(p) \times U(q)$ is a direct product, we obtain:

Lemma 8.9. The kernel of $T_{p, q}$ on $\mathbb{C}\left[S_{n}\right]$ is spanned by the elements $\pi E_{S}^{+}$with $|S|>p$ and by the elements $\pi E_{S}^{-}$with $|S|>q$.

Remark. The point is that $\pi E_{S}^{+}$can be used to reduce invariants of $U(p)$, while $\pi E_{S}^{-}$can be used to reduce invariants of $U(q)$. Since every invariant of $U(p) \times U(q)$ can be expressed in terms of the invariants of the respective factors, we are done.

Even in the cases of interest, however, $(q=p$ or $q=p+1)$, it is not clear how to use these relations to eliminate hyperoctahedral permutations with long decreasing subsequences; in particular, the invariants do not span the kernel of $T_{p, q}$ on $\mathbb{Z}\left[S_{n}\right]$ in general, but only on $\mathbb{Z}[1 / 2]\left[S_{n}\right]$. Similar remarks apply to $\otimes$. As in the other involution cases, the invariant space for $\nabla$ is associated to a (twisted) Gelfand pair in $H_{2 n}$; the relevant subgroup of $H_{2 n}$ is the centralizer of an element of $S_{n}^{\bigotimes}$. (For $\square$ and $\nabla$, the Gelfand pair is $\left(S_{2 n}, H_{n}\right.$ ), twisted in the $\nabla$ case.)

We observe that in each case, $\operatorname{ker}\left(T_{l}^{\circledast}\right) \subset \operatorname{ker}\left(T_{l-1}^{\circledast}\right)$ for all $l$, and $\operatorname{ker}\left(T_{n}^{\circledast}\right)=0$. We thus obtain the following theorem, which is a formal analogue of the Szegö limit theorem:

Theorem 8.10. We have the following limits of formal power series:

$$
\begin{aligned}
\lim _{l \rightarrow \infty} E_{U \in U(l)} \operatorname{det}\left(H(U ; x / y) H\left(U^{\dagger} ; z / w\right)\right) & =\prod_{j, k}\left(1-x_{j} z_{k}\right)^{-1}\left(1-y_{j} w_{k}\right)^{-1} \prod_{j, k}\left(1+x_{j} w_{k}\right)\left(1+y_{j} z_{k}\right) . \\
\lim _{l \rightarrow \infty} E_{U \in O(l)} \operatorname{det}(H(U ; x / y)) & =\prod_{j, k}\left(1+x_{j} y_{k}\right) \prod_{j \leq k}\left(1-x_{j} x_{k}\right)^{-1} \prod_{j<k}\left(1-y_{j} y_{k}\right)^{-1} . \\
\lim _{l \rightarrow \infty} E_{U \in S p(2 l)} \operatorname{det}(H(U ; x / y)) & =\prod_{j, k}\left(1+x_{j} y_{k}\right) \prod_{j<k}\left(1-x_{j} x_{k}\right)^{-1} \prod_{j \leq k}\left(1-y_{j} y_{k}\right)^{-1} .
\end{aligned}
$$

More precisely, all coefficients of degree $\leq 2 l$ agree, and each limit is monotonic in all coefficients.

Remark 1. Under the homomorphism $p_{j}(x / y) \mapsto f_{i}, p_{j}(z / w) \mapsto g_{i}$, we obtain

$$
\begin{aligned}
\lim _{l \rightarrow \infty} E_{U \in U(l)} \exp \left(\sum_{j} f_{j} \operatorname{Tr}\left(U^{j}\right) / j+\sum_{j} g_{j} \operatorname{Tr}\left(U^{-j}\right) / j\right) & =\exp \left(\sum_{j} f_{j} g_{j} / j\right) \\
\lim _{l \rightarrow \infty} E_{U \in O(l)} \exp \left(\sum_{j} f_{j} \operatorname{Tr}\left(U^{j}\right) / j\right) & =\exp \left(\sum_{j}\left(f_{j}^{2}+f_{2 j}\right) / 2 j\right) \\
\lim _{l \rightarrow \infty} E_{U \in S p(2 l)} \exp \left(\sum_{j} f_{j} \operatorname{Tr}\left(U^{j}\right) / j\right) & =\exp \left(\sum_{j}\left(f_{j}^{2}-f_{2 j}\right) / 2 j\right)
\end{aligned}
$$

and again coefficients with (weighted) degree $\leq 2 l$ agree. This fact was proved via representation theory in 10 (note that the degree bounds given there are incorrect); the connection to Szegö's limit theorem was observed in 23. The monotonicity result is new. 
Remark 2. There are, of course, also hyperoctahedral analogues, both of which simply reduce to the $\square$ case.

We can also give a partial extension of the above results to the "super" analogues of the classical groups. For the unitary supergroup $U(l / k)$, the centralizer algebra $C_{n}(U(l / k))$ is again spanned by permutations, under a particular representation $T_{l / k}$ of $S_{n}$.

Theorem 8.11. The representations that appear in $T_{l / k}$ with positive multiplicity are those indexed by partitions $\lambda$ with $\lambda_{l+1}<k+1$.

We then have:

Theorem 8.12. For any nonnegative integers $l$ and $k$ and compositions $\nu$ and $\nu^{\prime}$, the dimension of the space $\Pi\left(\nu^{\prime}\right) C_{n}(U(l / k)) \Pi(\nu)$ is equal to the number of multisets $M \in \mathcal{M}_{W W^{\prime}}\left(\nu ; \nu^{\prime}\right)$ such that

$$
\lambda_{W W^{\prime}}(M)_{l+1}<k+1 .
$$

Proof. If $T_{\lambda}$ is the representation of $S_{n}$ corresponding to the partition $\lambda$, then the dimension of

$$
T_{\lambda}\left(\Pi\left(\nu^{\prime}\right) S_{n} \Pi(\nu)\right)
$$

is given by the number of pairs of bitableau of shape $\lambda$ with respective content $\nu$ and $\nu^{\prime}$. In other words, by the generalized Knuth correspondence, this is equal to the number of multisets $M \in \mathcal{M}_{W W^{\prime}}^{\square}\left(\nu ; \nu^{\prime}\right)$ with

$$
\lambda_{W W^{\prime}}(M)=\lambda .
$$

The result follows by summing over $\lambda$.

Remark 1. The obvious conjecture that those multisets give a basis under $T_{l / k}$ is not true in general (not even for $C_{4}(U(1 / 1))$ ); thus Theorem 8.4 does not immediately extend to the supergroup case. It is also not clear whether there is a simple description of the kernel of $T_{l / k}$.

Remark 2. This theorem can be thought of as a formal statement along the lines of:

$$
E_{U \in U(l / k)} \operatorname{det}\left(H(U ; x) H\left(U^{\dagger} ; y\right)\right)=\sum_{\lambda_{l+1}<k+1} s_{\lambda}(x) s_{\lambda}(y) .
$$

It would be nice to make this statement precise. It is also interesting to speculate on the possibility of a "super" analogue of orthogonal polynomials and Toeplitz determinants.

Similarly, for the orthosymplectic supergroup $O S p(l / 2 k)$ (the full group, not just the component of the identity), the invariant space $F_{n}(O S p(l / 2 k))$ is the image of fixed-point-free involutions under a map $T_{l / 2 k}^{\square}$ for which

$$
T_{l / 2 k}(\pi) T_{l / 2 k}^{\square}(\tau)=T_{l / 2 k}^{\square}\left(\pi^{-1} \tau \pi\right) .
$$

This thus induces an action of $S_{2 n}$ on $F_{2 n}(O(l / 2 k))$, for which: 
Theorem 8.13. $F_{2 n}(O S p(l / 2 k))$ splits into $S_{n}$-irreducible submodules as the direct sum

$$
\bigoplus_{\substack{\lambda \vdash n \\ \lambda l+1 \leq k}} T_{2 \lambda} .
$$

It then follows that

Theorem 8.14. For any nonnegative integers $l$ and $k$ and compositions $\nu$ and $\nu^{\prime}$, the dimension of the space $\Pi(\nu) F_{2 n}(O S p(l / 2 k))$ is equal to the number of multisets $M \in \mathcal{M}_{W W^{\prime}}^{\square}(\nu ; 0)$ such that

$$
\lambda_{W W^{\prime}}(M)_{l+1}<2 k+1 \text {. }
$$

In "integral" form, this reads:

$$
E_{U \in O S p(l / 2 k)} \operatorname{det}(H(U ; x))=\sum_{\lambda_{l+1} \leq k} s_{2 \lambda}(x) .
$$

\section{References}

[1] J. Baik. Random vicious walks and random matrices. Comm. Pure Appl. Math., to appear. e-print math.PR/0001022.

[2] J. Baik and E. M. Rains. The asymptotics of monotone subsequences of involutions. e-print math.CO/9905084.

[3] J. Baik and E. M. Rains. Limiting distributions for a polynuclear growth model with external sources. $J$. Statist. Phys., to appear. e-print math.PR/0003135.

[4] A. Berele and A. Regev. Hook Young diagrams with applications to combinatorics and to representations of Lie superalgebras. Adv. in Math., 64(2):118-175, 1987.

[5] A. Berele and J. B. Remmel. Hook flag characters and their combinatorics. J. Pure Appl. Algebra, $35(3): 225-245,1985$.

[6] A. Borodin, A. Okounkov, and G. Olshanski. Asymptotics of Plancherel measures for symmetric groups. J. Amer. Math. Soc., 13(3):481-515, 2000. e-print math.CO/9905032.

[7] R. Brauer. On algebras which are connected with the semisimple continuous groups. Ann. of Math., 38(4):857-872, 1937.

[8] W. H. Burge. Four correspondences between graphs and generalized Young tableaux. J. Combin. Theory Ser. A, 17:12-30, 1974 .

[9] N. G. de Bruijn. On some multiple integrals involving determinants. J. Indian Math. Soc. (N.S.), 19:133$151,1955$. 
[10] P. Diaconis and M. Shahshahani. On the eigenvalues of random matrices. J. Appl. Probab., 31A:49-62, 1994.

[11] P. Forrester and E. Rains. Inter-relationships between orthogonal, unitary and symplectic matrix ensembles. e-print solv-int/9907008.

[12] E. R. Gansner. Matrix correspondences of plane partitions. Pacific J. Math., 92(2):295-315, 1981.

[13] I. Gessel. Symmetric functions and P-recursiveness. J. Combin. Theory Ser. A, 53:257-285, 1990.

[14] B. Gordon. Notes on plane partitions, V. J. Combin. Theory, 11:157-168, 1971.

[15] B. Gordon and L. Houten. Notes on plane partitions II. J. Combin. Theory, 4:81-99, 1968.

[16] I. P. Goulden. A linear operator for symmetric functions and tableaux in a strip with given trace. Discrete Math., 99:69-77, 1992.

[17] M. Grassl, M. Rötteler, and T. Beth. Computing local invariants of qubit systems. Phys. Rev. A, 58:1833$1839,1998$.

[18] C. Greene. An extension of Schensted's theorem. Adv. in Math., 14:254-265, 1974.

[19] F. D. Grosshans, G.-C. Rota, and J. A. Stein. Invariant Theory and Superalgebras, volume 69 of $C M B S$ Regional Conference Series in Mathematics. AMS, Providence, R.I., 1987.

[20] M. Ishikawa, S. Okada, and M. Wakayama. Applications of minor-summation formula I. Littlewood's formulas. J. Algebra, 183:193-216, 1996.

[21] M. Ishikawa and M. Wakayama. Minor summation formula of Pfaffians. Linear and Multilinear Algebra, 39:285-305, 1995.

[22] M. Jimbo. A $q$-analogue of $U(\mathfrak{g} l(N+1))$, Hecke algebra, and the Yang-Baxter equation. Lett. Math. Phys., 11(3):247-252, 1986.

[23] K. Johansson. On random matrices from the compact classical groups. Ann. of Math., 145(3):519-545, 1997.

[24] K. Johansson. Shape fluctuations and random matrices. Comm. Math. Phys., 209(2):437-476, 2000. e-print math.CO/9903134.

[25] D. E. Knuth. Permutations, matrices and generalized Young tableaux. Pacific J. Math., 34:709-727, 1970.

[26] D. E. Knuth. Sorting and Searching, volume 3 of The Art of Computer Programming. Addison Wesley, Reading, Mass., second edition, 1973.

[27] C. Krattenthaler. Identities for classical group characters of nearly rectangular shape. J. Algebra, 209:1-64, 1998. 
[28] B. Leclerc and J.-Y. Thibon. The Robinson-Schensted correspondence, crystal bases, and the quantum straightening at $q=0$. Electron. J. Combin., 6(2):R11, 1996.

[29] I. G. Macdonald. Symmetric Functions and Hall Polynomials. Oxford Univ. Press, Oxford, England, second edition, 1995.

[30] A. M. Odlyzko, B. Poonen, H. Widom, and H. S. Wilf. On the distribution of longest increasing subsequences in random permutations. manuscript in preparation.

[31] S. Okada. Applications of minor summation formulas to rectangular-shaped representations of classical groups. J. Algebra, 205:337-367, 1998.

[32] E. M. Rains. A mean identity for longest increasing subsequence problems. e-print math.CO/0004082.

[33] E. M. Rains. Increasing subsequences and the classical groups. Electron. J. Combin., 5(1):R12, 1998.

[34] A. Regev. The representations of $S_{n}$ and explicit identities for P.I. algebras. J. Algebra, 51:25-40, 1978.

[35] J. B. Remmel. The combinatorics of $(k, l)$-hook Schur functions. In C. Greene, editor, Combinatorics and Algebra, volume 34 of Contemporary Mathematics, pages 253-287, Providence, R.I., 1984. AMS.

[36] G.-C. Rota and B. Sturmfels. Introduction to invariant theory in superalgebras. In D. Stanton, editor, Invariant Theory and Tableaux, volume 19 of The IMA Volumes in Mathematics and its Applications, pages 1-35, New York, 1990. Springer-Verlag.

[37] D. W. Stanton and D. E. White. A Schensted algorithm for rim hook tableaux. J. Combin. Theory Ser. A, 40:211-247, 1985.

[38] J. R. Stembridge. Nonintersecting paths, pfaffians, and plane partitions. Adv. in Math., 83:96-131, 1990.

[39] G. Szegö. Orthogonal Polynomials, volume 23 of American Mathematical Society, Colloquium Publications. AMS, Providence, R.I., fourth edition, 1975.

[40] E. Thoma. Die unzerlegbaren, positiv-definiten Klassenfunktionen der abzählbar unendlichen symmetrischen Gruppe. Math. Z., 85:40-61, 1964.

[41] C. Tracy and H. Widom. Correlation functions, cluster functions and spacing distributions for random matrices. J. Statist. Phys., 92:809-835, 1998.

[42] M. van Leeuwen. The Robinson-Schensted and Schützenberger algorithms, an elementary approach. Electron. J. Combin., 3(2):R15, 1996.

[43] K. Vo and R. Whitney. Tableaux and matrix correspondences. J. Combin. Theory Ser. A, 35:328-359, 1983. 